\title{
Design and Fabrication of Bioinspired Hierarchical Dissipative Elastic Metamaterials
}

\author{
Marco Miniaci, ${ }^{1}$ Anastasiia Krushynska, ${ }^{2}$ Antonio S. Gliozzi, ${ }^{3}$ Nesrine Kherraz, ${ }^{4}$ Federico Bosia, ${ }^{4}$ and \\ Nicola M. Pugno ${ }^{2,5,6, *}$ \\ ${ }^{1}$ School of Aerospace Engineering and School of Mechanical Engineering, Georgia Institute of Technology, North \\ Avenue, Atlanta, Georgia 30332, USA \\ ${ }^{2}$ Laboratory of Bio-Inspired \& Graphene Nanomechanics, Department of Civil, Environmental and Mechanical \\ Engineering, University of Trento, Via Mesiano, 77, 38123 Trento, Italy \\ ${ }^{3}$ Department of Applied Science and Technology, Politecnico di Torino, Corso Duca degli Abruzzi 24, 10124 \\ Torino, Italy \\ ${ }^{4}$ Department of Physics and Nanostructured Surfaces and Interfaces Centre, University of Turin, Via Pietro \\ Giuria 1, 10125 Torino, Italy \\ ${ }^{5}$ School of Engineering \& Materials Science, Queen Mary University of London, Mile End Road, London E1 4NS, \\ $U K$ \\ ${ }^{6}$ KET Labs, Edoardo Amaldi Foundation, Italian Space Agency, Via del Politecnico snc, 00133 Rome, Italy
}

(Received 11 June 2016; revised manuscript received 26 May 2018; published 10 August 2018)

Hierarchical structures with constituents over multiple length scales are found in various natural materials like bones, shells, spider silk and others, all of which display enhanced quasistatic mechanical properties, such as high specific strength, stiffness, and toughness. At the same time, the role of hierarchy on the dynamic behavior of metamaterials remains largely unexplored. This study numerically and experimentally assesses the effect of bioinspired hierarchical organization as well as of viscoelasticity on the wave attenuation properties of continuous elastic metamaterials. We consider single-phase metamaterials formed by self-similar unit cells with different hierarchical levels and types of hierarchy. Two types of structures are considered: a hub-spoke geometry with thin connecting elements and nested hierarchical organization, and a crosslike porous geometry with external hierarchical organization. In the first, hierarchical elements occur at similar size scales, while in the second they differ by one order of magnitude. Results highlight a number of advantages through the introduction of structural hierarchy. Band gaps relative to the corresponding nonhierarchical structures are mostly preserved in both types of structures, but additional hierarchically-induced band gaps also appear, and the hierarchical configuration allows the tuning of band-gap frequencies to lower frequencies in the crosslike porous geometry, with a simultaneous significant reduction of the global structural weight. We show that even small viscoelastic effects are essential in determining the overall attenuation behavior, including between band gaps. Finally, we verify the numerically-predicted multifrequency band gaps by experimental characterization of the transmission properties of crosslike structures. The approach we propose allows the incorporation of hierarchical organization in existing metamaterial configurations, with the corresponding improvement of wave-damping properties, thus extending application possibilities for elastic metamaterials to multiple frequency scales.

DOI: 10.1103/PhysRevApplied.10.024012

\section{INTRODUCTION}

Biological structural materials are renowned for their exceptional mechanical characteristics, often surpassing synthetic high-performance materials [1]. Spider silk, bone, enamel, limpet teeth are examples of materials that combine high specific strength and stiffness with outstanding toughness and flaw resistance [2-4]. Many studies have shown hierarchical structure to be responsible for these properties, e.g., providing many energy dissipation

\footnotetext{
*nicola.pugno@unitn.it
}

and crack deflection mechanisms over various size scales to contribute to high toughness [5]. However, studies in biomechanics linking material structure to function have mainly been limited to the quasistatic regime while the dynamic properties of these materials have been somewhat less investigated although notable examples of impact tolerance (e.g., the bombardier beetle's explosion chamber [6]) or vibration damping (e.g., the woodpecker skull [7]) have been observed.

A systematic study of the dynamic properties of composite structures such as those found in biomaterials can exploit analysis tools and methods widely applied in 
the field of elastic metamaterials. These are periodically arranged composite structures with advanced dynamic functionalities, e.g., the ability to generate band gaps (BGs, i.e., frequency ranges where the wave propagation is inhibited), wave focusing or collimation, negative refraction, and defect states [8]. These features make elastic metamaterials attractive for various applications, including seismic wave shielding [9-11], noise abatement [12,13], vibration isolation [14], acoustic cloaking [15], nondestructive evaluation [16], subwavelength imaging [17], and even thermal management [18]. In the past two decades, the mechanism of BG nucleation (Bragg scattering [19] and/or local resonance [20]) has been widely studied at various spatial and frequency scales. Conventional configurations are mostly based on elementary unit cells comprising single inclusions or cavities, which results in limited BG frequency ranges [8,21-24]. Optimized design of the unit cells has allowed the size of a single BG or even several BGs to be maximized, e.g., by using topology optimization or multiobjective optimization methods [25-27]. More sophisticated metamaterial architectures with compliant elements, exploiting thermally coupled dissipation mechanisms, have also been proposed but have demonstrated limited attenuation performance at broadband frequencies due to the strong dependence on the structural thickness that prevents the design of stiff, lightweight configurations suitable for engineering applications [28]. Therefore, lightweight, practically oriented metamaterial structures with multiscale wave attenuation abilities remain an open field of research.

One way to potentially overcome this limitation is to exploit fractal-like and bioinspired structural hierarchy. The latter is here understood in the sense that a representative unit cell comprises multiple arrangements of inhomogeneities at various size scales. If the same arrangement occurs at every size scale, the pattern is self-similar. If a unit cell as a whole is geometrically equivalent to its parts at any hierarchical level, the structure is fractal-like [29].

Several recent studies have reported hierarchical and fractal-like metamaterials with superior wave attenuation in broadband frequency ranges due to their structural organization [29-34]. It has been shown that hierarchical lattice-type metamaterials are characterized by multiple BGs with advanced load-carrying capabilities [32], improved thermal resistance [30], and a hybrid type of BG generation mechanism [29]. Similarly, continuous heterogeneous hierarchical metamaterials have demonstrated broadband vibration mitigation capabilities combined with improved mechanical performance [35]. In these studies, the total structural weight is preserved when introducing each new hierarchical level. Although some advantages of incorporating structural hierarchy in metamaterials have been highlighted for lattice and heterogeneous configurations, the introduction of hierarchy in single-phase continuous metamaterials remains to be investigated. These hierarchical designs are mostly inspired by biological materials, e.g., the wellknown brick and mortar microstructure [35], nacre and bone microstructures $[33,36]$, the biocalcite architecture [31], etc. A great variety of further hierarchical patterns can be found in natural structures, such as in butterfly wings, wood, leafs, macaw feathers, grass stems, kelp, corals, cotton, human bone, cuttlefish bone, diatoms and sponges [37,38]. All these patterns are characterized by a porous structure and can thus be exploited for bioinspiration, providing an alternative approach to that adopted so far, which has implied the conservation of the structural mass when introducing additional hierarchical levels. Porous hierarchical structures, on the other hand, allow the advantages of hierarchical organization to be fully exploited for the design of lightweight and robust continuous metamaterials.

In this work, we therefore propose to introduce hierarchy in continuous single-phase metastructures with cavities by reducing the structural weight - an approach that has not been analyzed so far - when introducing an additional hierarchical level. The key idea is to incorporate hierarchically organized sets of cavities into regular metamaterials with the design goal of efficiently extending their BGs to multiple frequency ranges [39]. The resulting porouslike structures are more lightweight than the regular case. This way of introducing hierarchy can be easily applied to new or existing metastructures with cavities, making it advantageous for practical applications. As regular configurations, we consider two metamaterial geometries, defined as hub-spoke or crosslike cavities, which are known for their ability to generate wide BGs [40,41]. For each geometry, we study a different type of hierarchical organization, namely a so-called nested hierarchy, when hierarchical structures of a subsequent level are located inside the previous level, and an external hierarchy, when hierarchical structures are located externally to those of the previous level. Additionally, we provide a detailed analysis of the possibilities provided by hierarchical organization as a function of the spatial ratio of hierarchical levels, and explicitly evaluate the critical effect of material viscosity. As in previous works $[30-33,35,36]$, here the hierarchical metamaterial design follows a bioinspired approach, meaning that without necessarily emulating the dynamic properties of any specific biological system, we draw inspiration from the structures found in porous hierarchical materials and use their organizational principles to create novel metamaterial models.

\section{MODELS AND METHODS}

Biological hierarchically structured materials occur in a great variety of microstructural patterns [37]. In many 
cases, the structures are heterogeneous, typically with reinforcing (stiffer) elements such as fibers, platelets, or crystals embedded in a softer, often amorphous matrix [1]. In other cases (such as in bone), voids, cavities, or canals are also included, contributing to reduced mass density, but also potentially to effects on elastic wave propagation or damping [7]. Hierarchical Levels (HL) often span various length scales, from nanometers to centimeters [1]. In other cases, hierarchical structures occur on similar size scales, as in the case of wood, leafs, grass stems, corals, or sponges [37,38]. Despite the great variety of hierarchical structures occurring in nature, distinctive features can be observed and extracted in order to drive and inspire the design of elastic metamaterials with enhanced dynamic properties. Representative structures can be found, e.g., in diatom cell walls or frustules (Fig. 1). Two typical hierarchical configurations are a nested arrangement of smaller scale objects inside a larger scale frame [Fig. 1(a)] or, in contrast, the distribution of smaller cavities around larger ones [Fig. 1(b)]. Additionally, many biological microstructures contain high-aspectratio (slender) constitutive elements [such as in Fig. 1(a)], which can play a key role in altering their vibrational characteristics.

Based on these patterns, we study metamaterial configurations with (i) thin connecting elements and a nested hierarchical organization, defined here as a hub-spoke geometry [Fig. 1(a)] and (ii) crosslike cavities with external hierarchical organization, referred to as crosslike porous structures [Fig. 1(b)]. The crosslike geometry of the cavities in case (ii) is chosen due to its proven efficiency in inducing larger BGs compared to other geometries of the same cavity volume [40,42]. In both cases, the hierarchical organization is achieved introducing (or repeating) one (or several) self-similar structures obtained by scaling the starting geometry by a hierarchical factor (HF). Notice that this type of structure provides an advantage with respect to other hierarchical structures considered in the literature, such as in [33], since samples can be easily fabricated through a recursive procedure, whereby successive hierarchical levels can be introduced incrementally by adding nested cavities internally or externally with respect to the existing structure.

The representative unit cells of the structures are shown in Fig. 1 with the geometric parameters given in Table I. In both cases, two HLs of self-similar patterns are considered. The hub-spoke geometry is used to investigate hierarchical elements having similar size scales, i.e., comparable dimensions of the unit cells: $a_{1}=0.4 a_{2}$ [Fig. 1(a)]. The crosslike porous geometry is used to investigate hierarchical elements having an order of magnitude difference in spatial scale: $a_{1}=0.05 a_{2}$ [Fig. 1(b)]. These designs can naturally be extended to $n$ levels of hierarchy.

Hierarchical metamaterials are composed by infinitely duplicating the unit cells in Fig. 1 in a periodic 2D array.
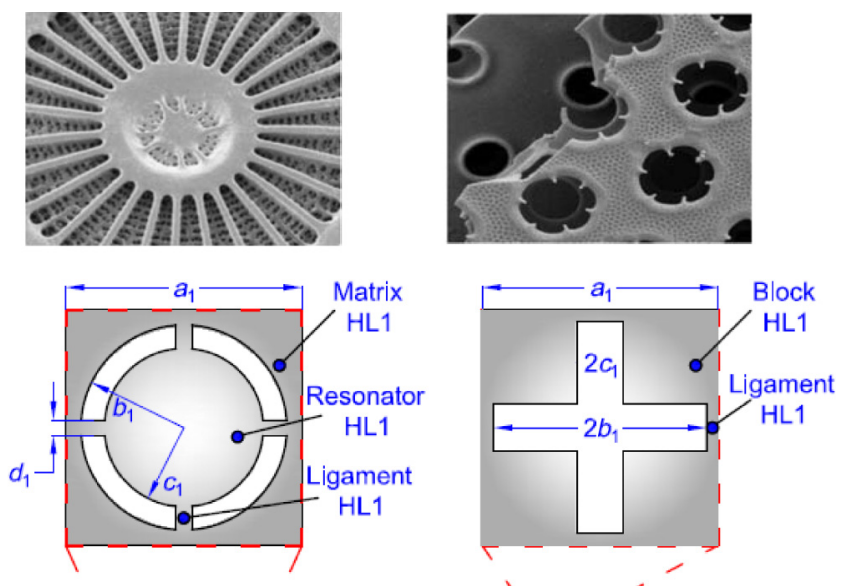

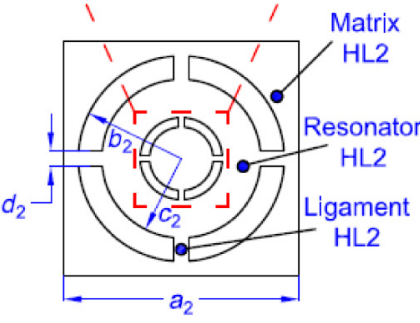

(a)

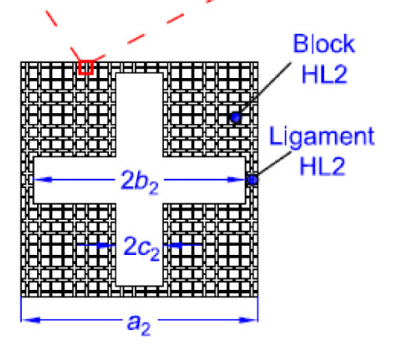

(b)
FIG. 1. Hierarchical porous structures found in natural materials at different spatial scales (in this example in diatom cell walls $[58,59])$ and the corresponding bioinspired hierarchical metamaterial unit cells: (a) structures with thin connecting elements and nested hierarchical organization (hub-spoke geometry) and (b) crosslike cavities and external hierarchical organization (crosslike porous metamaterial).

The out-of-plane dimension of the unit cells is assumed to be much larger compared to the lateral ones, allowing a 2D plane strain approximation to be adopted [43,44]. A typical polymeric material such as those used in 3D printing is considered for the structures. Specifically, we use the mechanical properties of the thermoplastic polymer acrylonitrile butadiene styrene (ABS), assuming isotropic linear elasticity: bulk modulus $K=3.34 \mathrm{GPa}$, shear modulus $G=0.714 \mathrm{GPa}$, and mass density $\rho=1050 \mathrm{~kg} / \mathrm{m}^{3}$ [45]. Viscoelastic dissipation is also taken into account, by assuming attenuation linearly proportional to frequency, typical for many polymeric materials [46]. For monochromatic waves, complex-valued elastic constants can then be

TABLE I. Case studies and geometrical parameters used in the simulations. HF is a hierarchical factor indicating the ratio between hierarchical levels $n$ and $n+1$, i.e., $\mathrm{HF}=a_{n} / a_{n+1}$.

\begin{tabular}{lccccc}
\hline \hline & & \multicolumn{4}{c}{ Parameters (m) } \\
\cline { 3 - 6 } Case study & $\mathrm{HF}$ & $a_{n}$ & $b_{n}$ & $c_{n}$ & $d_{n}$ \\
\hline Hubspoke & 0.4 & $4 \times 10^{-4}$ & $0.45 a_{n}$ & $0.35 a_{n}$ & $0.05 a_{n}$ \\
Crosslike porous & 0.05 & $1 \times 10^{-3}$ & $0.45 a_{n}$ & $0.15 a_{n}$ & - \\
\hline \hline
\end{tabular}


written using a Kelvin-Voigt viscoelastic model [47]:

$$
K_{\mathrm{ve}}=K+i \omega \theta_{\mathrm{loss}}, G_{\mathrm{ve}}=G+i \omega \eta_{\mathrm{loss}},
$$

where the subscript ve indicates the viscoelastic case, $\omega$ is the angular frequency, and $\theta_{\text {loss }}, \eta_{\text {loss }}$ are the viscosity levels.

Contrary to previous approaches in the literature [29, 34], here the introduction of additional HLs implies an equivalent density reduction, since the aim is to realize lightweight structures with optimized attenuating properties compared to their heavier nonhierarchical counterparts. For the hub-spoke structure, the density reduction is less than $5 \%$ compared to the nonhierarchical structure, while for the crosslike porous metamaterial, it reaches $45 \%$.

The description of the formalism adopted to model wave propagation in the considered periodic structures, both in the elastic and viscoelastic cases, is presented in the Supplemental Material [48], as well as its implementation in finite-element (FE) simulations to derive dispersion and transmission spectra [19,45-48].

\section{LINEAR ELASTIC HIERARCHICAL METAMATERIAL}

\section{A. Hierarchical organization at similar spatial scales}

Figure 2 shows dispersion diagrams for the hub-spoke metamaterial [Fig. 1(a)] with both regular (nonhierarchical) and hierarchical organization. The size of the two representative unit cells $a_{2}$ is maintained constant, in order to analyze the effects of hierarchy on the wave dispersion in the same frequency range. Unit-cell optimization is discussed in the Supplemental Material for the regular and hierarchical structures [48]. Direct comparison of the diagrams [Figs. 2(a) and 2(b)] reveals two important advantages of hierarchical organization: (i) partial conservation of the BGs originating from the regular geometry and (ii) nucleation of new BGs in the midfrequency range.

The BGs are said to be partially preserved because the introduction of hierarchy has a twofold effect: it alters some of the pre-existing dispersion curves and it induces new pass bands, resulting in a possible modification of the original BGs. Physically, this happens because the introduction of the hierarchical architecture alters the stiffnessmass ratio and introduces additional degrees of freedom (at each iteration, a new matrix, a new resonator, and new connectors linking the two are added to the system). As a result, new vibration modes appear [e.g., see Figs. 2(c)-2(f)].

The additional bands introduced due to the hierarchical organization can exhibit nonlocalized or localized behavior. In the first case, the flat portions of the curves corresponding to localized motions (either at the internal HL1 resonator level [Fig. 2(c)] or at the ring-like resonator level
[Figs. 2(d) and 2(e)]). In the second case, portions of the curves appear with positive or negative slope, involving the combined vibration of both structures occurring in the hierarchical geometry [Fig. 2(f)]. Since these curves can be located inside or outside a preexisting $\mathrm{BG}$ at the previous HL, they can cause the BG split into two or more BGs [compare gray rectangles in Figs. 2(a) and 2(b)].

To gain insight into the nature of the BGs nucleated due to the hierarchy, we first analyze the BG mechanisms in the original, nonhierarchical structure. For this purpose, we evaluate a midgap Bragg scattering frequency from the condition of structural periodicity, i.e., $f_{B G}=c_{p} / 2 a$ with $c_{p}$ indicating the effective phase velocity in the medium. For the unit cell with slender elements, the effective velocity can be evaluated directly from the band-structure diagram as $c_{p}=\omega / k$ in the vicinity of the $\Gamma$ point and is indicated by the slope of tangents to the fundamental longitudinal and shear modes [dotted lines in Fig. 2(a)] as proposed in Ref. [49]. The intersection of a tangent with the Brillouinzone boundary at point $X$ provides the value of $f$ BG for each mode type. The midgap Bragg frequency of the longitudinal mode falls within the first (lowest) BG. Thus, this BG is generated due to the Bragg-scattering effect of longitudinal waves in the slender connectors [see also Figs. $2(\mathrm{~g})$ and 2(h)]. The location of the second BG at approximately twice the frequency of the first and the similarity of the mode shapes at the bounds of the two BGs [compare Figs. 2(g) and 2(h) and Figs. 2(l)-2(o)] suggest that the second BG is also induced by the Bragg scattering of higher-frequency waves.

The additional pass bands in the band diagram of the hierarchically structured unit cell [Fig. 2(b)] decrease the width of the first BG. At the same time, hierarchy leads to the change in the mass-stiffness ratio of the resonator at HL1 level and introduces another set of slender elements, which, in turn, cause the generation of the two other BGs at the mid- and high-frequency ranges. The two new BGs are defined "hierarchically induced" and are highlighted by red to distinguish them from the original BGs. Although the BG formation mechanism is different, this result is reminiscent of the hybrid BGs obtained in [29] with the introduction of hierarchical structure in a rather different system, i.e. beam lattices. The new BGs are generated due to the Bragg scattering of longitudinal waves in the connectors of HL2 level. This conclusion also follows from the analysis of vibration modes. For example, one observes that the pattern at the lower bound of the second BG for the nonhierarchical structure [Fig. 2(1)] is exactly the same as that at the lower bound of the second additional BG [Fig. 2(d)]. Therefore, the BGs found in the metamaterials with a nested hierarchy are the superposition of the Bragg BGs for longitudinal waves in slender elements at different hierarchical levels. The comparison of the band structures in Figs. 2(a) and 2(b) highlights another feature of hierarchical organization: the similarity of dispersion curves below 


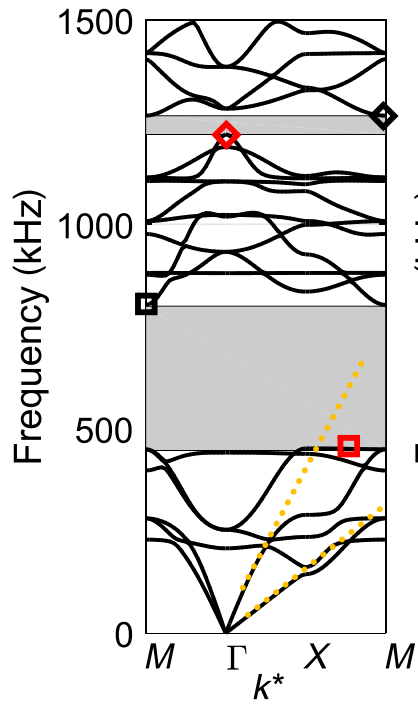

(a)

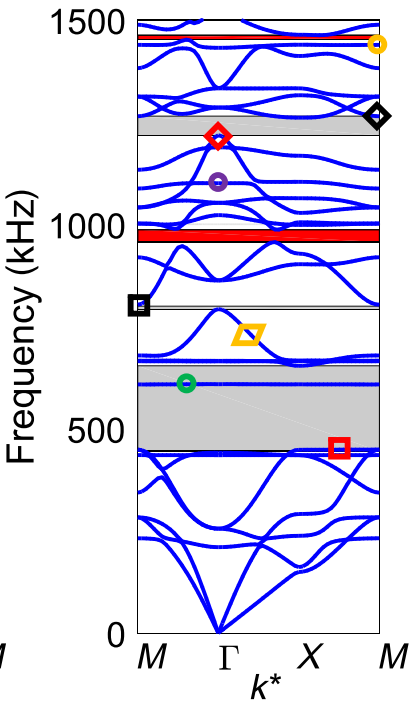

(b)

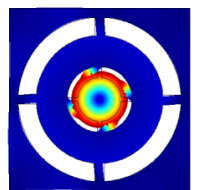

$\circ(\mathrm{c})$
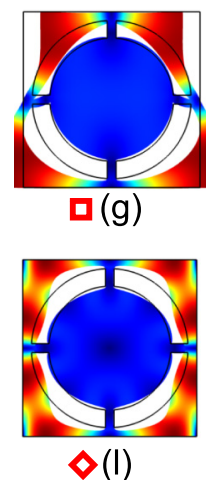

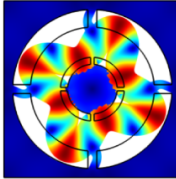

$\circ(\mathrm{d})$

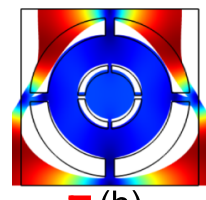

口(h)

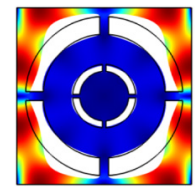

$\diamond(\mathrm{m})$

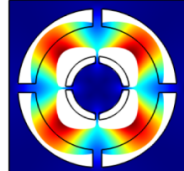

$\circ(\mathrm{e})$

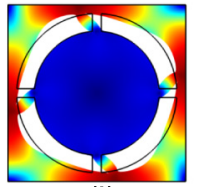

口(j)

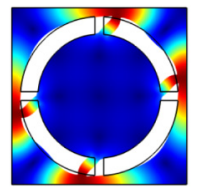

$\diamond(n)$

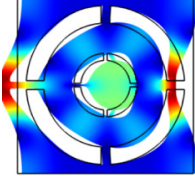

$\square$ (f)

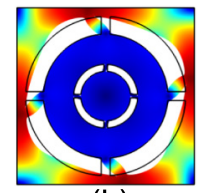

$\mathbf{\square}(\mathrm{k})$

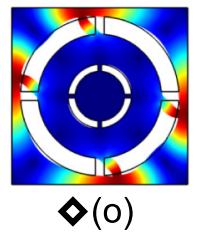

FIG. 2. Dispersion diagrams for the hub-spoke metamaterial with: (a) regular and (b) hierarchical organization. The size of the two representative unit cells is maintained constant and equal to $a_{2}$, in order to analyze the effects of hierarchy on the wave dispersion in the same frequency range. Ordinary BGs are indicated in gray. Hierarchical structure allows additional BGs at mid and high frequencies to be induced (highlighted in red). (c)-(o) Vibration patterns at various frequencies. Colored symbols indicate their corresponding location on the band diagrams. The intersection of the dashed lines with the Brillion-zone boundary at point $X$ indicate approximate mid-Bragg scattering frequencies for the fundamental longitudinal and shear modes.

the first BG for both structures. This occurs since lowfrequency waves (below $250 \mathrm{kHz}$ for the analyzed case) are insensitive to the absence of small portions of the material in the unit cell due to the introduction of hierarchy. By varying the $\mathrm{HF}$ value, one increases or decreases the size of these elements, and thus, can tune the BG frequencies and width as shown in Fig. 3. For instance, by decreasing the HF from 0.42 to 0.38 , it is possible to obtain up to approximately three times larger BGs [see Figs. 3(a)-3(c)] as well as to localize the motion at the level of the internal ring, limiting the deformations in the HL2 matrix (see Fig. 3d).

Furthermore, hierarchical organization can be useful to isolate dispersion curves with negative group velocity [see Figs. 3(a)-3(c)], which makes hierarchically organized metamaterials attractive for potential applications exploiting negative refraction [50,51].

\section{B. Hierarchical organization at different spatial scales}

Next, we analyze wave dispersion in a crosslike porous metamaterial with an external hierarchical organization at different spatial scales [Fig. 1(b)]. This type of design has been optimized for large BG formation in previous works $[40,52]$. The band structures for the regular and hierarchical metamaterial configurations are shown in Figs. 4(a) and 4(b), respectively. It is apparent that the wide BG ranging from approximately 300 to $740 \mathrm{kHz}$ in Fig. 4(a) is almost completely preserved in the diagram of Fig. 4(b). Pass bands located inside the latter BG are represented by mostly flat lines corresponding to localized modes [Fig. 4(c)]. As discussed below, these modes become evanescent in real structures, which always have a certain level of energy dissipation.

Figure 5 shows the dispersion diagrams for the unit cell of a regular metamaterial with a lattice parameter of the same size of $a_{2}$ in the HL2 [Fig. 5(a)] and hierarchical metamaterial [Fig. 5(b)] in the low-frequency range. The analysis is similar to that in Section III A and reveals that the $\mathrm{BG}$ in the regular structure is generated due to the Bragg-scattering mechanism of longitudinal waves in the slender parts of the regular metamaterial unit cell, since the midgap Bragg frequency is located within the lowest BG. The introduction of hierarchy results in the shift of this BG to approximately three times lower frequencies and the opening of several other BGs at the BG frequencies of the regular structure. These other BGs are also of Braggtype, induced by the changes in the mass-stiffness ratios for the unit-cell elements. Again, due to the self-similarity of the constitutive geometries at different HLs, the dispersion curves below the lowest BG have similar trends for hierarchical and nonhierarchical structures, together with the corresponding vibration patterns [Figs. 5(c) and 5(d)].

To explain the shift of the first BG to lower frequencies, we consider an equivalent mass-spring model capable of predicting the BG bounds for a metamaterial with crosslike holes [40]. According to this model, a square lattice of crosslike cavities can be considered as a lattice of square blocks connected by thin ligaments (see Fig. SM6(a) in 


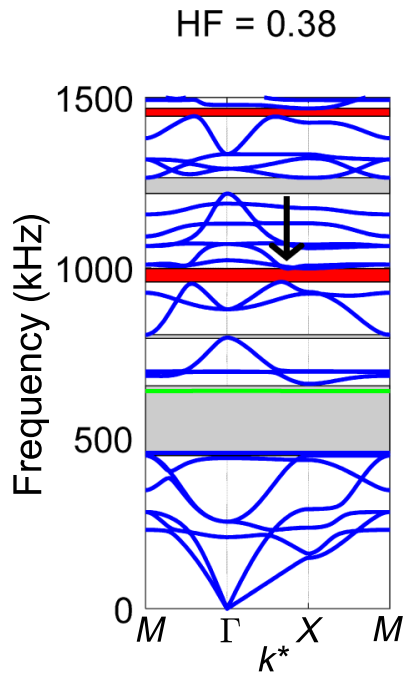

(a)

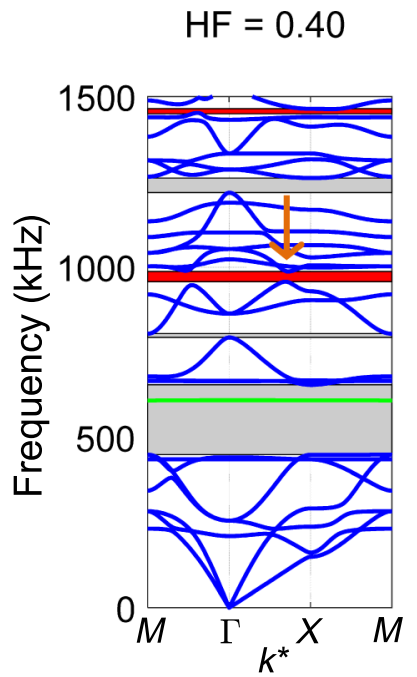

(b)

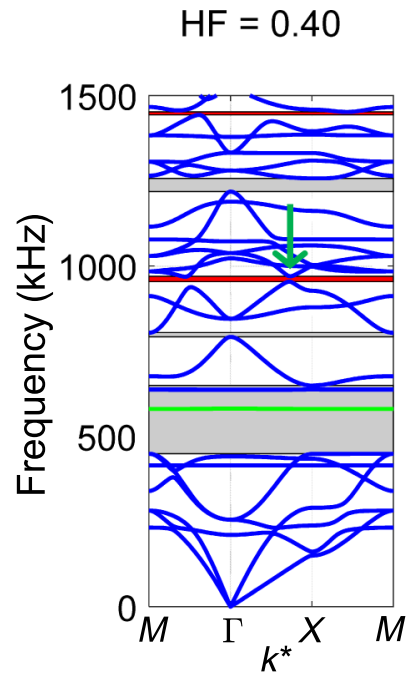

(c)

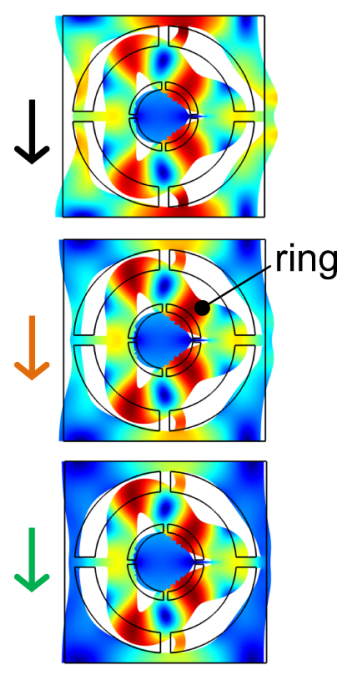

(d)

FIG. 3. Dispersion diagrams for the hub-spoke metamaterial for three different values of the HF: (a) 0.38, (b) 0.40, and (c) 0.42. (d) Mode shapes at the edges of the hierarchically induced BGs.

the Supplemental Material [48]). At low frequencies, the blocks behave as rigid bodies, whereas ligaments play the role of springs (Fig. SM6b). Thus, the lower bound of the BG is evaluated as [40]

$$
\omega_{l}^{\mathrm{BG}}=\sqrt{\frac{\bar{K}}{\bar{M}}},
$$

where $\bar{K}=2 S\left\{\left[(\lambda+2 \mu) /\left(l_{b}+l_{s}\right)\right]+\left(\alpha \mu / l_{s}\right)\right\}$ and $\bar{M}=$ $m_{l}+\left(m_{s} / 2\right)$ are effective stiffness and effective mass, respectively; $m_{b}, m_{s}$ and $l_{b}, l_{s}$ are masses and lengths of

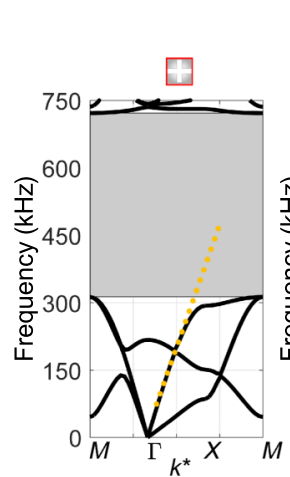

(a)

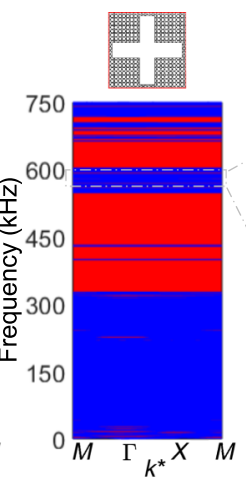

(b)

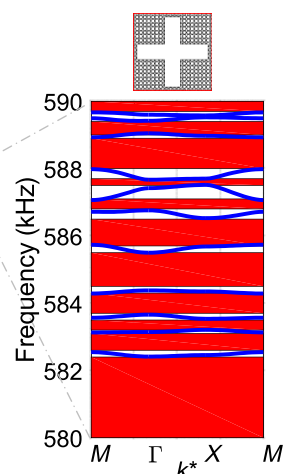

(c)
FIG. 4. Dispersion diagrams for the crosslike porous metamaterial with (a) regular and (b) external hierarchical organization. The size for the unit cell of the regular structure is the same as that for hierarchical level 1. (c) A part of the dispersion diagram for a hierarchical metamaterial inside the BG. The hierarchical organization at different spatial scales allows the BG originating from a smaller constitutive geometry to be preserved. the block (subscript $b$ ) and springs (subscript $s$ ); $S$ is the cross section of the springs, $\lambda=K-(2 / 3) G$ and $\mu=G$ are the Lamé constants, and $\alpha$ is a weighting coefficient.

The same model can be applied to the self-similar hierarchical structure. Obviously, by introducing the hierarchy, the lengths of the blocks and ligaments remain unchanged. Therefore, for the calculation of the effective stiffness $\bar{K}^{h}$, the only variable quantity is the cross section $S_{1}^{h}=\left(a_{1}-\right.$ $\left.2 b_{1}\right) \times 1$, which is $S_{1}^{h}=\mathrm{HF} \times S_{2}^{h}$, with $S_{2}^{h}$ indicating the cross section of the structure at HL2 (superscript $h$ refers to a hierarchical structure). As mentioned previously, the mass of the hierarchical unit cell is $45 \%$ of the regular counterpart, i.e., $\bar{M}^{h}=0.45 \bar{M}^{r}$ (superscript $r$ refers to a regular structure). The ratio of the lower bounds for the band gaps in the hierarchical and regular structures is then as follows:

$$
\frac{\omega^{h}}{\omega^{r}}=\sqrt{\frac{\bar{K}^{h} \bar{M}^{r}}{\bar{M}^{h} \bar{K}^{r}}}=\sqrt{\frac{0.05}{0.45}}=\frac{1}{3} .
$$

Therefore, the equivalent spring-mass model adapted for a hierarchical structure with crosslike holes predicts a shift of the lower BG bound to three times lower frequencies, while the calculated data gives $\omega^{h} / \omega^{r}=1 / 2.82$, i.e., the accuracy of the model approximation is $94 \%$. Hence, the first BG in the band structure of the hierarchical unit cell is generated by the same mechanism as in a regular unit cell, and its shift to lower frequencies occurs due to the reduction of the effective stiffness and mass of the slender parts. Similar shifts can be achieved by exploiting hierarchical organization of non-self-similar structures. However, selfsimilarity of hierarchical constituents enables similarity to 


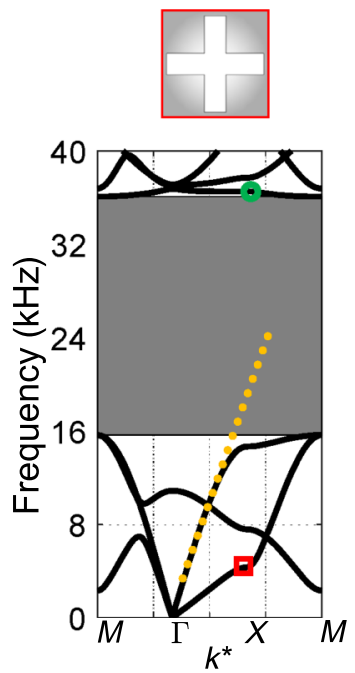

(a)

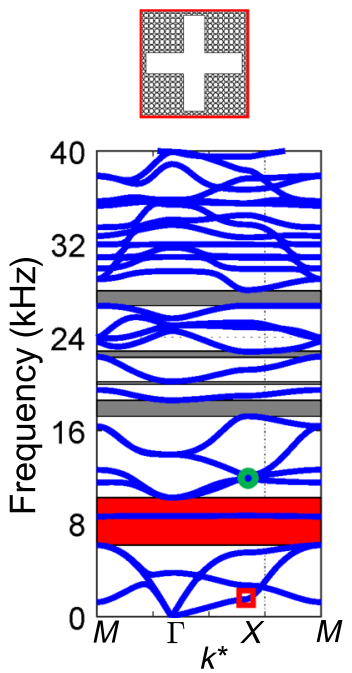

(b)

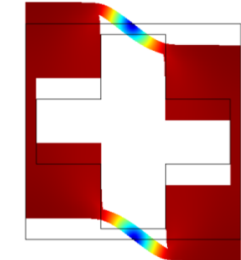

(c)

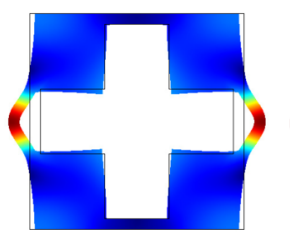

o (e)

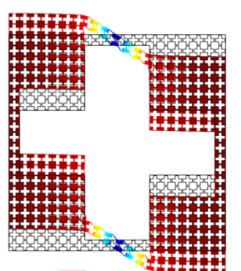

(d)

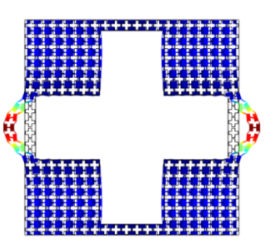

O (f)
FIG. 5. Dispersion diagrams for the crosslike porous metamaterial with (a) regular and (b) hierarchical organization. The size for the regular unit cell is the same as for HL 2. The hierarchical organization allows the first $\mathrm{BG}$ to be shifted to lower frequencies. (c)-(f) Vibration patterns for the regular and hierarchical unit cells. The intersection of the dashed line with the Brillouin-zone boundary at point $X$ indicates an approximate mid-Bragg scattering frequency for the fundamental longitudinal mode. be preserved in the dispersion-curve trends below the lowest BG. Above the BG, the curve trends differ, but the vibration patterns in the vicinity of the $\mathrm{BG}$ are still similar [see Figs. 5(e) and 5(f)].

Overall, the introduced external hierarchical organization can be considered as a means of tuning BGs to lower frequencies accompanied by the simultaneous reduction of the structural weight and generation of additional BGs at higher frequencies. This mechanism may extend applicability ranges of elastic metamaterials and facilitate their wide practical utilization. Moreover, the main advantages found for the nested hierarchical organization at similar spatial scales are preserved for the metamaterial with the external hierarchy at different spatial scales. Specifically, these are (1) (partial) conservation of the BGs induced by the constitutive geometries, (2) nucleation of additional BGs in the midfrequency range, and (3) similarity of the dispersion curves below the $\mathrm{BG}$ frequencies for the selfsimilar hierarchical structure and the regular counterpart. These properties are common to various hierarchical structures, as demonstrated in the Supplemental Material, where other designs are considered with similar results [48].

\section{DISSIPATIVE HIERARCHICAL METAMATERIALS}

Many materials, e.g., polymers used to produce 3Dprinted samples, typically display wave-dissipation characteristics. For solid structures, this can be taken into account by considering viscoelastic properties of the metamaterial constituents [53]. Viscoelastic material behavior usually influences wave-propagation characteristics of elastic metamaterials and modifies band-structure diagrams calculated under assumption of the linear elasticity [47].

To analyze viscoelastic effects on wave dispersion in hierarchically organized metamaterials, we assume dissipation to be linearly proportional to frequency, which is admissible for most polymers [46,53]. Since, under isothermal conditions, the bulk modulus of most polymers appears to retain a stationary equilibrium value over a range of time scales $[47,53,54]$, i.e., $K_{\mathrm{ve}}=K$ and $\theta_{\text {loss }}=0$ in Eq. (1), time dependency of the shear modulus $G$ can be effectively exploited. In the frequency domain, viscoelastic dissipation is then described by the Kelvin-Voigt model in Eq. (1), which approximates the response of real polymers well [47] and is commonly used to study damped metamaterials $[47,55]$.

To model physically realistic scenarios, the values of shear viscosity $\eta_{\text {loss }}$ are chosen by considering the viscoelastic effect on the wave dispersion in a homogeneous material, similarly to [47]. It is found that in the considered frequency range for $\eta_{\text {loss }} \leq 5 \mathrm{Pas}$, the real part of wave vectors for propagating modes is almost unchanged compared to that for the corresponding elastic case, while the imaginary part shows small deviations from zero values at higher frequencies, up to $800 \mathrm{kHz}$. Hence, these values correspond to small material dissipation [47,56]. Considering that the objective of this study is not to model wave dissipation in any specific polymer, but rather to theoretically analyze the influence of viscoelasticity on the dynamics of hierarchically organized metamaterials, we choose the representative values of $\eta_{\text {loss }}=2 \mathrm{Pas}$ and $\eta_{\text {loss }}=5$ Pa s to obtain indicative results.

For a viscoelastic medium, pure propagating waves or BGs can no longer be identified, since all waves are attenuating or evanescent. Thus, the study is performed 
(a)

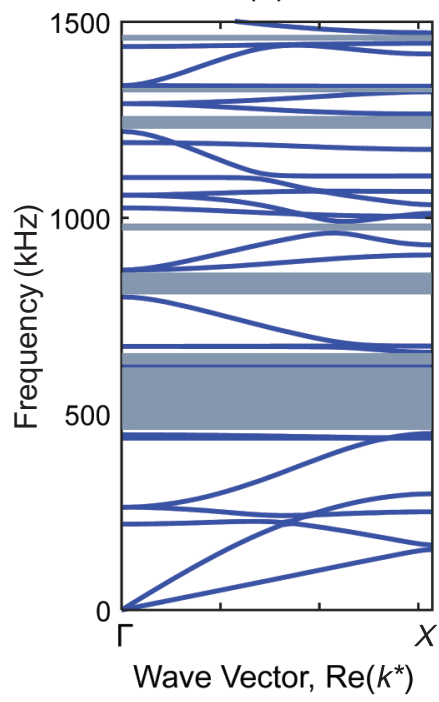

(b)

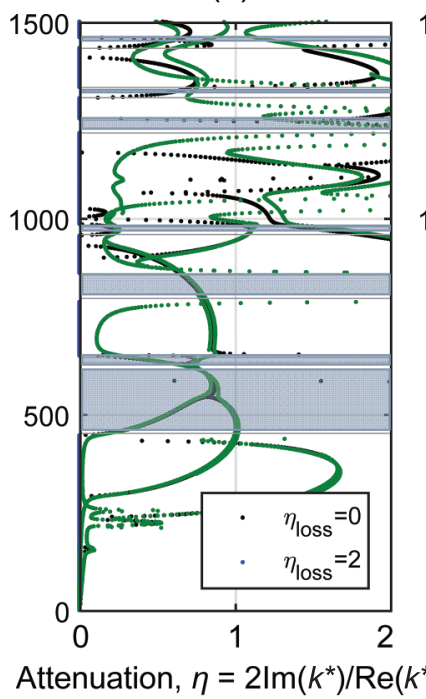

(c)

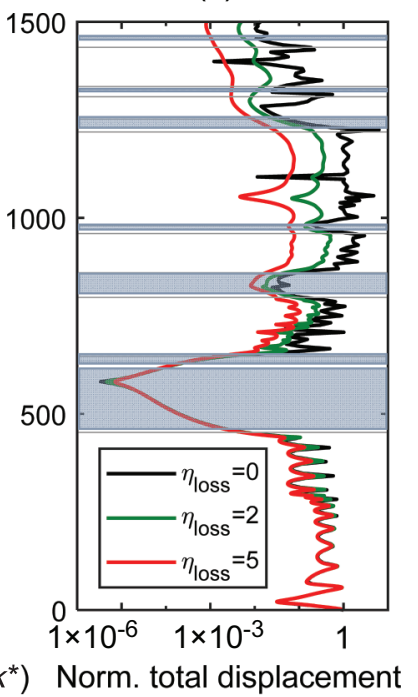

FIG. 6. (a) Dispersion diagram for waves propagating in $\Gamma-X$ direction in the hub-spoke metamaterial with hierarchical organization. (b) Attenuation spectrum for the same metamaterial with linear elastic $(K, G)$ and viscoelastic $\left(K_{\mathrm{ve}}=K, G_{\mathrm{ve}}=\right.$ $G+i \omega \eta_{\text {loss }}, \eta_{\text {loss }}=2$ Pa s) material behavior. (c) Transmission spectrum for elastic and viscoelastic hierarchical metamaterials. Propagating elastic waves are shown by blue, attenuating elastic and viscoelastic waves are shown by black, dark green $\left(\eta_{\text {loss }}=2\right.$ Pa s $)$, and red ( $\eta_{\text {loss }}=5$ Pas), respectively. Viscoelasticity leads to the expected frequencyproportional increase of the wave attenuation with curve-rounding trends near the BG bounds. for experimentally realistic scenarios, for which waves are characterized by real-valued frequencies and complexvalued wave vectors $\mathbf{k}=\operatorname{Re} \mathbf{k}+i \operatorname{Im} \mathbf{k}$ with the imaginary part $\operatorname{Im} \mathbf{k}$ describing the wave spatial attenuation. The intensity of the wave attenuation can be evaluated by considering either an attenuation spectrum relating the frequency to the wave attenuation level $2 \operatorname{Im}(\mathbf{k}) / \operatorname{Re}(\mathbf{k})[47,57]$ or a transmission spectrum showing amplitudes of transmitted waves normalized with respect to the amplitude of the input signal, versus the wave frequency.

Figure 6(a) shows a dispersion diagram for waves propagating in the $\Gamma-X$ direction in the hub-spoke hierarchical metamaterial together with attenuation [Fig. 6(b)] and transmission [Fig. 6(c)] spectra for elastic and viscoelastic $\left(\eta_{\text {loss }}=2 \mathrm{Pas}\right.$ and $\left.\eta_{\text {loss }}=5 \mathrm{Pas}\right)$ cases. In the latter, the transmission coefficient is defined as the normalized total displacement, i.e., the amplitude of the total displacements averaged over the evaluated area $\left(\sqrt{u_{\text {out }}^{2}+v_{\text {out }}^{2}}\right)$ divided by the amplitude of the input signal $\left(u_{\text {in }}\right)$. Figure 6(a) reproduces a part of Fig. 2(b) for only one propagation direction. The BGs for the elastic structure are highlighted by shaded regions. According to the definition, the wave attenuation for propagating modes $(\operatorname{Im} \mathbf{k}=0)$ in the elastic case is zero outside the BGs and has finite values for the evanescent modes $(\operatorname{Im} \mathbf{k} \neq 0)$. The attenuation diagram of waves shown in Fig. 6(b) is in good agreement with their transmission characteristics for both elastic and viscoelastic structures, discussed below. Despite the small level of viscosity, wave attenuation and transmission in the viscoelastic metamaterial differs from that in its elastic counterpart starting from frequencies of the lowest BG. In general, the attenuation (transmission) increases (decreases) linearly with the frequency, in agreement with the adopted assumption. Viscoelasticity at most influences the BG bounds, where straight edges of attenuation curves, typical for the elastic case, become rounded in a similar way as for regular metamaterials $[47,56]$. No peculiar influence of viscoelasticity is observed for the additional BGs opened due to hierarchy. These effects are similar for the two considered viscoelastic values, confirming that the derived conclusions are of a general nature.

Similar features are found for waves propagating in the crosslike porous metamaterial with external hierarchy at different spatial scales. Here, we focus our attention on frequencies within the BGs generated by crosses of HL1. Figure 7(a) shows a part of the dispersion diagram given in Fig. 4(c) for waves propagating in the $\Gamma-X$ direction in the specified frequency range. Results show that there are many (almost) flat pass bands forming the dispersion spectrum preventing the formation of a complete BG at the considered frequencies. Poor wave-attenuation performance of the metamaterial is also found in the transmission spectrum [Fig. 7(c)], where multiple peaks appear in correspondence with these pass bands (black line). However, when material dissipation is taken into account, all the pass bands are transformed into attenuating waves with nonzero imaginary part values, as emerges comparing the attenuation spectra for the elastic (black lines) and viscoelastic (dark green lines, $\eta_{\text {loss }}=2 \mathrm{~Pa} \mathrm{~s}$ ) metamaterial models in Fig. 7(b). The smallest attenuation level for the viscoelastic case exceeds the maximum attenuation in the elastic case. Also, the introduction of viscoelasticity leads to a considerable modification of the attenuation spectrum. All of the almost flat pure real bands become complex-valued with a large imaginary part [Fig. 7(b)]. Interestingly, the maximum attenuation occurs at lower 
(a)

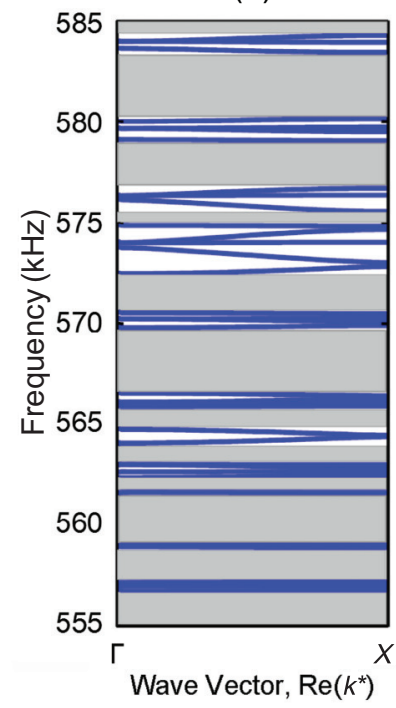

(b)

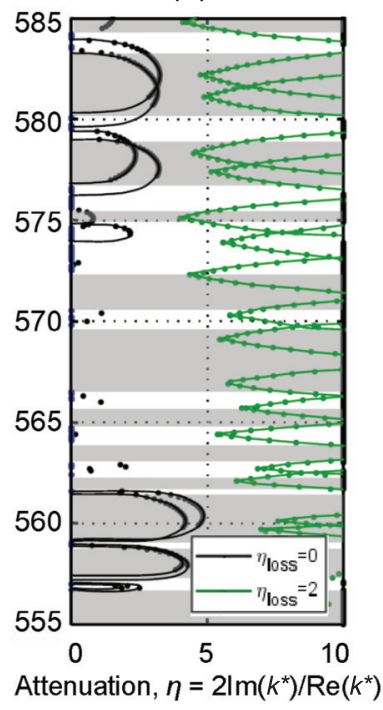

(c)

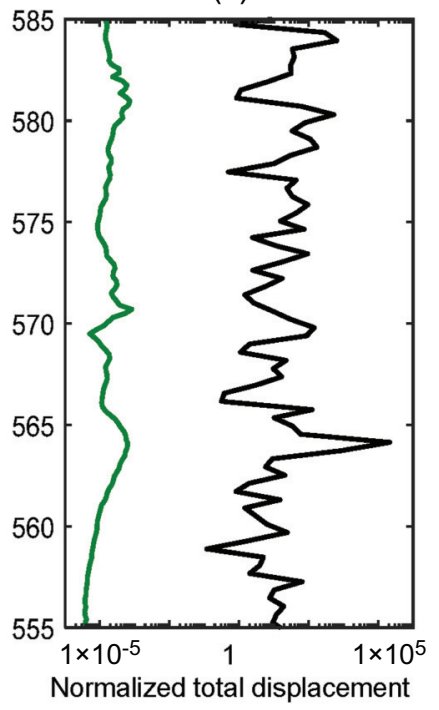

FIG. 7. (a) Dispersion diagram for waves propagating in $\Gamma-X$ direction in the crosslike porous metamaterial with hierarchical organization. (b) Attenuation spectrum for the same metamaterial with linear elastic $(K, G)$ and viscoelastic $\left(K_{\mathrm{ve}}=\right.$ $K, G_{\mathrm{ve}}=G+i \omega \eta_{\text {loss }}, \eta_{\text {loss }}=2$ Pa s) material behavior. (c) Transmission spectrum for the same metamaterial with elastic $(K, G$; black $) \quad$ and viscoelastic $\quad\left(K_{\mathrm{ve}}=K, G_{\mathrm{ve}}=\right.$ $G+2 i \omega$; green) material behavior. frequencies, apparently contradicting the assumption that attenuation is linearly increasing with frequency. The reason for this is that the modes near $555 \mathrm{kHz}$ form the upper bound of a large BG for the elastic metamaterial, where the curve-rounding effect occurs [56]. The same conclusion can be derived from the analysis of the transmission spectra for the viscoelastic case [dark green curve in Fig. 7(c)]. For the same model, as in the elastic case considered above, the transmission level is very low in the whole frequency range, indicating a complete BG. Similar results are obtained for other BG frequencies of the hierarchical structure. As the viscosity level increases from 2 to $5 \mathrm{Pas}$, the transmission coefficient becomes very small, indicating that this level of viscosity is too high for the considered frequencies.

In summary, we can conclude that BGs generated in structures of a lower hierarchical level are preserved for hierarchically organized metamaterials, provided that material losses are taken into account. Hence, the mathematical model accounting for wave dissipation is not only more realistic, but also enables advanced wave-attenuation performance of the metamaterial generated by the interplay between BG generation mechanisms and the attenuation characteristics of the material constituents to be revealed.

\section{EXPERIMENTAL CHARACTERIZATION}

To experimentally confirm the capability of the structure to nucleate BGs at different frequency scales, we consider a hierarchical metamaterial of the type discussed in Sec. III B, consisting of crosslike cavities at two different spatial scales. The specimen consists of a $10-\mathrm{mm}$-thick and 960-mm-long $\times 120$-mm-large aluminum plate with an array of six hierarchical crosslike cavities machined in its central portion. The sample is obtained via waterjet cutting starting from a unique pristine aluminum plate, exhibiting linear elastic properties with the following nominal mechanical parameters $\rho=2700 \mathrm{~kg} / \mathrm{m}^{3}, E=70 \mathrm{GPa}$ and $v=0.33$. The specimen is represented schematically in Fig. 8(a) and dimensions are provided in the Supplemental Material in Fig. SM9 [48].

We perform two sets of transmission experiments through the structure, in order to characterize its frequency-dependent transparency and/or its partial or total BGs. These fall in low- or high-frequency ranges, depending on the relation of the wavelengths with the characteristic length scales of the structure (large or small crosslike cavities, respectively). Experiments on the aluminum sample are performed using two different excitation systems in the low- and high-frequency ranges. In the first case, we excite wave propagation in the sample using a frequency sweep from 1 to $12 \mathrm{kHz}$ (with a sweep rate of $500 \mathrm{~ms}$ ), by means of an amplified vibration exciter (TIRA, Shaker 51110) glued to the lower surface of the plate, in its homogeneous portion [see Fig. 8(b)]. The signal is recorded after the propagation through the hierarchical

(a)

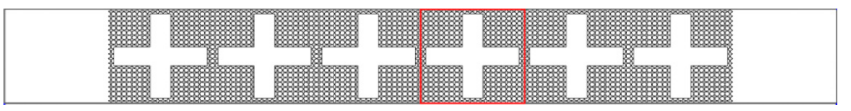

(b)

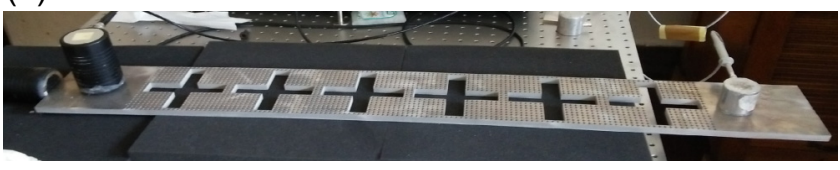

FIG. 8. (a) Schematic of the experimental specimen and (b) sample with applied piezoelectric transducers for signal generation and detection. Refer to SM [48] for the geometrical dimensions of the specimen. 
(a)

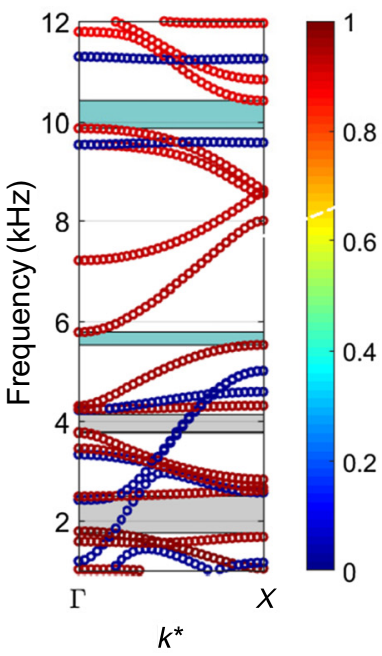

(c)

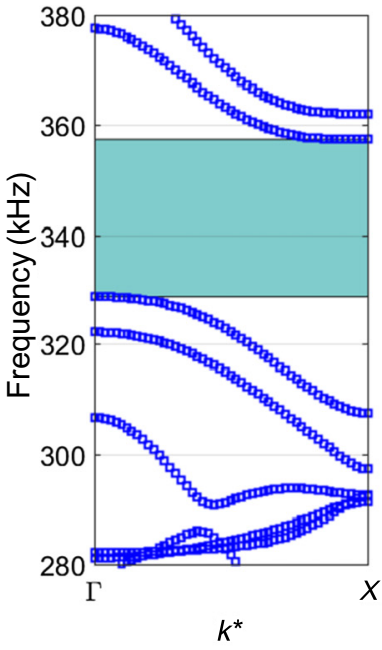

(b)

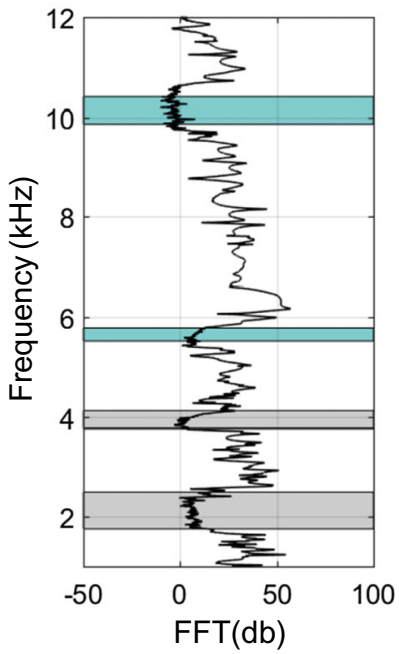

(d)

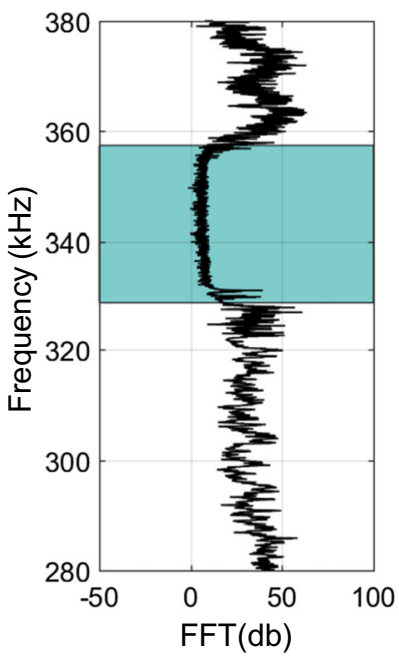

FIG. 9. Comparison between numerical predictions and experimentally measured transmitted signals in the low- and highfrequency ranges. (a) Numerical dispersion spectrum in the low-frequency range. Curve color indicates mode polarization, from red to blue; (b) experimental FFT of the transmitted signal in the low-frequency range; (c) numerical dispersion spectrum in the high-frequency range; and (d) experimental FFT of the transmitted signal in the high-frequency range.

metamaterial by two acquisition systems in order to capture different vibrational modes and distinguish between partial and total BGs in the transmission spectrum. The first acquisition system is a scanning laser vibrometer (Polytec, OFV 505) placed orthogonally to the surface, recording out-of-plane vibrations. The second acquisition system is a broadband piezotransducer sensor glued on top of the surface of the sample. No significant difference is observed between the two acquisition methods and only the one using a piezoelectric transducer has been reported for the sake of clarity. High-frequency excitation, on the other hand, is induced by a traditional piezotransducer

sweeping from 280 to $380 \mathrm{kHz}$, glued to the same region as the shaker used for low-frequency excitation. The acquisition is performed in the same way as previously described (laser and piezotransducer). The spectral response obtained in transmission experiments is compared to the dispersion diagrams obtained by FE analysis and results are shown in Fig. 9. The numerically predicted modes in the low-frequency range $(1-12 \mathrm{kHz})$ for the $\Gamma X$ direction are shown in Fig. 9(a), with curve color indicating mode polarization, from blue for in-plane (0 in the scale bar) to red for out-of-plane (1 in the scale bar). Partial and total BGs are indicated by gray and green rectangles, respectively. Figure 9(b) illustrates the fast Fourier transform (FFT) of the measured transmitted signal in the same frequency range. Results clearly show a very close agreement with numerical predictions, including the case of partial BGs, since the corresponding modes are not excited and detected because of the adopted excitation-detection setup. The uniform attenuation level within the BGs supports the claim that they are induced by the Bragg-scattering mechanism. The numerically calculated mode shapes are shown in the Supplemental Material (Fig. SM10) [48].

The numerically predicted high-frequency dispersion spectrum is shown in Fig. 9(c), displaying a single full BG centered at $345 \mathrm{kHz}$, confirmed by the experimental data in Fig. 9(d). This type of BG is generated by the small crosslike cavities in the hierarchical structure.

Thus, experiments confirm the effects predicted numerically and described previously, at least for structures with a large difference between hierarchical scales. In particular, the hierarchical structure preserves the band structure of the nonhierarchical structure at low frequency, but BGs are shifted to lower frequencies due to a reduction of the mean stiffness and density. At the same time, the smaller features of the hierarchical structure simultaneously provide BGs at higher frequencies.

\section{CONCLUSIONS}

We have numerically and experimentally investigated the influence of bioinspired hierarchical organization and material viscoelasticity on wave dispersion in metamaterials with self-similar constituents at various spatial scales. Contrary to previous approaches, our study focuses on porouslike hierarchical structures, whereby increasing hierarchy entails a weight reduction. Results reveal various advantages of the hierarchical structure on the dynamic performance of elastic metamaterial. These are as:

(1) conservation of most of the BGs induced by the constitutive regular geometries, in the presence of material damping;

(2) nucleation of additional hierarchically induced BGs in the midfrequency range; 
(3) similar wave dynamics at low frequencies for hierarchical and corresponding regular structures;

(4) shift of BGs to lower frequencies due to the effective mass and stiffness reduction.

We have discussed the dynamics for the occurrence of hierarchically induced BGs, and shown the generality of the observed behavior, which is not limited to a specific configuration. Additionally, experiments convincingly reproduce numerical predictions that an external structural hierarchy occurring at different spatial scales can be exploited to tune BGs of the regular metamaterial counterpart to lower frequencies while significantly reducing structural weight. A simple equivalent mass-spring model has been developed to predict this BG shift. We have analyzed the effect of material dissipation losses on wave propagation in hierarchical metamaterials, establishing their crucial role, even when they are small, on the preservation of the BG size. Future experiments will concentrate on the precise determination of the frequency regions where material viscoelasticity leads to more significant effects. Overall, this work provides insights on how the hierarchical organization can be exploited to alter the dynamics of elastic metamaterials and reveals that the principles of bioinspired hierarchy can lead to lightweight metamaterials with multiscale frequency attenuation properties, providing useful design guidelines for practical applications.

\section{ACKNOWLEDGMENTS}

A.K. acknowledges financial support from the Department of Department of Civil, Environmental and Mechanical Engineering, University of Trento. N.M.P. is supported by the European Commission H2020 under the Graphene Flagship Core 2 No. 785219 (WP14 Polymer Composites) and FET Proactive Neurofibres Grant No. 732344 as well as by the Italian Ministry of Education, University and Research (MIUR) under the "Departments of Excellence" grant L.232/2016. F.B. and N.K. are supported by H2020 FET Proactive Neurofibres Grant No. 732344 and by project Metapp (No CSTO160004) co-funded by Fondazione San Paolo.

[1] M. Meyers, P. Chen, A. Lin, and Y. Seki, Biological materials: Structure and mechanical properties, Prog. Mater. Sci. 53, 1 (2008).

[2] S. Cranford, A. Tarakanova, N. Pugno, and M. Buehler, Nonlinear material behaviour of spider silk yields robust webs, Nature 482, 72 (2012).

[3] H. Gupta, J. Seto, W. Wagermaier, P. Zaslansky, P. Boesecke, and P. Fratzl, Cooperative deformation of mineral and collagen in bone at the nanoscale, Proc. Natl Acad. Sci. USA 103, 17741 (2006).
[4] A. Barber, D. Lu, and N. M. Pugno, Extreme strength observed in limpet teeth, J. R. Soc. Interface 12, 20141326 (2015).

[5] R. O. Ritchie, The conflicts between strength and toughness, Nat. Mater. 10, 817 (2011).

[6] E. Arndt, W. Moore, W.-K. Lee, and C. Ortiz, Mechanistic origins of bombardier beetle (Brachinini) explosioninduced defensive spray pulsation, Science 348, 563 (2015).

[7] S.-H. Yoon and S. Park, A mechanical analysis of woodpecker drumming and its application to shock-absorbing systems, Bioinspir. Biomim. 6, 016003 (2011).

[8] P. E. Deymier, Acoustic Metamaterials and Phononic Crystals (Springer, Heidelberg, 2013).

[9] M. Miniaci, A. Krushynska, F. Bosia, and N. Pugno, Large scale mechanical metamaterials as seismic shields, New J. Phys. 18, 083041 (2016).

[10] S. Kroedel, N. Thome, and C. Daraio, Wide band-gap seismic metastructures, Ext. Mech. Lett. 4, 111 (2015).

[11] A. Colombi, D. Colquitt, P. Roux, S. Guenneau, and R. Craster, A seismic metamaterial: The resonant metawedge, Sci. Rep. 6, 27717 (2016).

[12] R. Martínez-Sala, J. Sancho, J. V. Sánchez, V. Gómez, J. Llinares, and F. Meseguer, Sound attenuation by sculpture, Nature 378, 241 (1995).

[13] F. Morandi, M. Miniaci, A. Marzani, L. Barbaresi, and M. Garai, Standardised acoustic characterisation of sonic crystals noise barriers: Sound insulation and reflection properties, Appl. Acoust. 114, 294 (2016).

[14] H. Sun, X. Du, and P. Pai, Theory of metamaterial beams for broadband vibration absorption, J. Intell. Mater. Syst. Struct. 21, 1085 (2010).

[15] D. Colquitt, M. Brun, M. Gei, A. Movchan, N. Movchan, and I. Jones, Transformation elastodynamics and cloaking for flexural waves, J. Mech. Phys. Solids 72, 131 (2014).

[16] M. Miniaci, A. S. Gliozzi, B. Morvan, A. Krushynska, F. Bosia, M. Scalerandi, and N. M. Pugno, Proof of Concept for an Ultrasensitive Technique to Detect and Localize Sources of Elastic Nonlinearity Using Phononic Crystals, Phys. Rev. Lett. 118, 214301 (2017).

[17] M. Molerón and C. Daraio, Acoustic metamaterial for subwavelength edge detection, Nat. Commun. 6, 8037 (2015).

[18] L. Yang, J. Chen, N. Yang, and B. Li, Significant reduction of graphene thermal conductivity by phononic crystal structure, Int. J. Heat Mass Transf. 91, 428 (2015).

[19] L. Brillouin, Wave Propagation in Periodic Structures (Dover Phoenix, New York, 1946).

[20] Z. Liu, X. Zhang, Y. Mao, Y. Zhu, Z. Yang, C. Chan, and P. Sheng, Locally resonant sonic materials, Science 289, 1734 (2000).

[21] M. Maldovan and E. Thomas, Periodic Materials and Interface Lithography: For Photonics, Phononics and Mechanics (Wiley-VCH, Weinheim, 2009).

[22] Y. Pennec, J. O. Vasseur, B. Djafari-Rouhani, L. Dobrzynski, and P. A. Deymier, Two-dimensional phononic crystals: Examples and applications, Surf. Sci. Rep. 65, 229 (2010).

[23] A. Krushynska, V. Kouznetsova, and M. Geers, Towards optimal design of locally resonant acoustic metamaterials, J. Mech. Phys. Solids 71, 179 (2014). 
[24] R. V. Craster and S. Guenneau, Acoustic Metamaterials: Negative Refraction, Imaging, Lensing and Cloaking (Springer, Heidelberg, 2013).

[25] M. I. Hussein, K. Hamza, G. M. Hulbert, R. A. Scott, and K. Saitou, Multiobjective evolutionary optimization of periodic layered materials for desired wave dispersion characteristics, Struct. Multidisc. Optim. 31, 60 (2006).

[26] H.-W. Dong, X.-X. Su, Y.-S. Wang, and C. Zhang, Topological optimization of two-dimensional phononic crystals based on the finite element method and genetic algorithm, Struct. Multidisc. Optim. 50, 593 (2014).

[27] W. Xu, W. Wang, and T. Yang, Multi-objective optimization of layered elastic metamaterials with multiphase microstructures, J. Vib. Acoust. 135, 041010 (2013).

[28] O. Umnova, K. Attenborough, and C. Linton, Effects of porous covering on sound attenuation by periodic arrays of cylinders, J. Acoust. Soc. Am. 119, 278 (2006).

[29] Q. Lim, P. Wang, S. Koh, E. Khoo, and K. Bertoldi, Wave propagation in fractal-inspired self-similar beam lattices, Appl. Phys. Lett. 107, 221911 (2015).

[30] Y. Chen, Z. Jia, and L. Wang, Hierarchical honeycomb lattice metamaterials with improved thermal resistance and mechanical properties, Compos. Struct. 152, 395 (2016).

[31] Y. Chen and L. Wang, Multiband wave filtering and waveguiding in bio-inspired hierarchical composites, Ext. Mech. Lett 5, 18 (2015).

[32] Y. Chen and L. Wang, Harnessing structural hierarchy to design stiff and lightweight phononic crystals, Ext. Mech. Lett. 9, 91 (2016).

[33] P. Zhang and A. To, Broadband wave filtering of bioinspired hierarchical phononic crystal, Appl. Phys. Lett. 102, 121910 (2013).

[34] D. Mousanezhad, S. Babaee, H. Ebrahimi, R. Ghosh, A. S. Hamouda, K. Bertoldi, and A. Vaziri, Hierarchical honeycomb auxetic metamaterials, Sci. Rep. 5, 18306 (2015).

[35] Y. Chen and L. Wang, Bio-inspired heterogeneous composites for broadband vibration mitigation, Sci. Rep. 5, 17865 (2015).

[36] Y. Chen and L. Wang, Tunable band gaps in bio-inspired periodic composites with nacre-like microstructure, J. Appl. Phys. 116, 063506 (2014).

[37] P. Fratzl and R. Weinkamer, Nature's hierarchical materials, Prog. Mater. Sci. 52, 1263 (2007).

[38] B.-L. Su, C. Sanchez, and X.-Y. Yang, Hierarchically Structured Porous Materials: From Nanoscience to Catalysis, Separation, Optics, Energy, and Life Science (WileyVCH, Weinheim, 2012).

[39] M. Miniaci, A. Krushynska, F. Bosia, and N. M. Pugno. Bio-inspired hierarchical dissipative metamaterials, arXiv:1606.03596.

[40] Y.-F. Wang, Y.-S. Wang, and X.-X. Su, Large bandgaps of two-dimensional phononic crystals with cross-like holes, J. Appl. Phys. 110, 113520 (2011).

[41] A. Krushynska, M. Miniaci, F. Bosia, and N. Pugno, Coupling local resonance with Bragg band gaps in singlephase mechanical metamaterials, Ext. Mech. Lett. 12, 30 (2017).
[42] M. Miniaci, A. Marzani, N. Testoni, and L. De Marchi, Complete band gaps in a polyvinyl chloride (PVC) phononic plate with crosslike holes: Numerical design and experimental verification, Ultrasonics 56, 251 (2015).

[43] P. Wang, F. Casadei, S. Shan, J. C. Weaver, and K. Bertoldi, Harnessing Buckling to Design Tunable Locally Resonant Acoustic Metamaterials, Phys. Rev Lett. 113, 014301 (2014).

[44] L. De Marchi, A. Marzani, and M. Miniaci, A dispersion compensation procedure to extend pulse-echo defects location to irregular waveguides, NDT E Int. 54, 115 (2013).

[45] See http://www.makeitfrom.com/material-properties/Acry lonitrile-Butadiene-Styrene-ABS.

[46] D. Royer and E. Dieulesaint, Elastic Waves in Solids (Wiley, New-York, 1999).

[47] A. Krushynska, V. Kouznetsova, and M. Geers, Viscoelastic effects on wave dispersion in three-phase acoustic metamaterials, J. Mech. Phys. Solids 96, 29, 2016.

[48] See Supplemental Material at http://ink.aps.org/supple mental/10.1103/PhysRevApplied.10.024012 for dispersion spectra in elastic and viscoelastic cases, parametric studies and alternative geometries, analytical approaches, and numerical analysis for experimental tests.

[49] K. H. Matlack, A. Bauhofer, S. Krödel, A. Palermo, and C. Daraio, Composite 3D-printed metastructures for lowfrequency and broadband vibration absorption, Proc. Natl Acad. Sci. USA 113, 8386 (2016).

[50] A. Sukhovich, L. Jing, and J. Page, Negative refraction and focusing of ultrasound in two-dimensional phononic crystals, Phys. Rev. B 77, 014301 (2008).

[51] J.-F. Robillard, J. Bucay, P. A. Deymier, A. Shelke, K. Muralidharan, B. Merheb, J. O. Vasseur, A. Sukhovich, and J. H. Page, Resolution limit of a phononic crystal superlens, Phys. Rev. B, 83, 224301 (2011).

[52] M. Miniaci, M. Mazzotti, M. Radzieński, N. Kherraz, P. Kudela, W. Ostachowicz, B. Morvan, F. Bosia, and N. Pugno, Experimental observation of a large low-frequency band gap in a polymer waveguide, Front. Mater. 5, 8 (2018).

[53] J. Ferry, Viscoelastic Properties of Polymers (John Wiley and Sons, New York, 1980).

[54] J. De Vreugd, Ph.D. thesis: Delft University of Technology, 2011.

[55] E. Andreassen and J. Jensen, Analysis of phononic bandgap structures with dissipation, ASME J. Vibr. Acoust. 135, 041015 (2013).

[56] R. Moiseyenko and 1. V. Laude, Material loss influence on the complex band structure and group velocity in phononic crystals, Phys. Rev. B, 83, 064301 (2011).

[57] L. Cremer, M. Heckl, and B. Petersson, Structure-Born Sound (Springer, Berlin Heidelberg, 2005).

[58] S. Nishinaga, Diatom photographs, http://fineartamerica. com/featured/2-diatom-alga-sem-susumu-nishinaga.html.

[59] A. Fossdal, Science News from Science Daily, The Norwegian University of Science and Technology (NTNU), http://www.sciencedaily.com/releases/2012/07/120717100 117.htm 


\section{Supplementary material for}

"Design and fabrication of bioinspired hierarchical dissipative elastic metamaterials"

Marco Miniaci ${ }^{1}$, Anastasiia Krushynska ${ }^{2}$, Antonio S. Gliozzi ${ }^{3}$, Nesrine Kherraz ${ }^{4}$, Federico Bosia ${ }^{4}$, Nicola M. Pugno $2,5,6, *$

${ }^{1}$ School of Aerospace Engineering and School of Mechanical Engineering, Georgia Institute of Technology, North Avenue, GA 30332 Atlanta, USA

${ }^{2}$ Laboratory of Bio-Inspired \& Graphene Nanomechanics, Department of Civil, Environmental and Mechanical Engineering, University of Trento, Via Mesiano, 77, 38123, Trento, Italy

${ }^{3}$ Department of Applied Science and Technology, Politecnico di Torino, Corso Duca degli Abruzzi 24, 10124, Torino, Italy.

${ }^{4}$ Department of Physics and Nanostructured Surfaces and Interfaces Centre, University of Turin, Via Pietro Giuria 1, 10125, Torino, Italy

${ }^{5}$ School of Engineering \& Materials Science, Queen Mary University of London, Mile End Road, London, E1 4NS, UK

${ }^{6}$ KET Labs, Edoardo Amaldi Foundation, Italian Space Agency, Via del Politecnico snc, 00133 Rome, Italy

*nicola.pugno@unitn.it 


\section{Evaluation of dispersion spectra: numerical procedure}

We consider small-amplitude vector waves in a linear elastic medium. In the absence of external forces the wave field is described by standard wave equations for two-dimensional displacements $\boldsymbol{u}(\boldsymbol{x}, t)=\left(u_{x}(x, y, t), u_{y}(x, y, t)\right)[1]:$

$$
G \nabla^{2} \boldsymbol{u}+\left(K+\frac{G}{3}\right) \nabla(\nabla \cdot \boldsymbol{u})=\rho \frac{\partial^{2} \boldsymbol{u}}{\partial t^{2}}
$$

where $\nabla$ is the gradient operator. The analysis is restricted to harmonic waves and forms a necessary basis for studying other types of wave motions. Due to the structural periodicity of the metamaterials, the displacements are also periodic in space and can be represented using the Floquet-Bloch expansion theorem [2] as follows:

$$
\mathbf{u}(\mathbf{x}, t)=\mathbf{U}(\mathbf{x}) e^{i \mathbf{k}^{T} \cdot \mathbf{x}} e^{i \omega t}
$$

where $\boldsymbol{U}(\boldsymbol{x})=\left(U_{x}(x, y), U_{y}(x, y)\right)$ is the Bloch wave amplitude of the same period as the structural lattice, $\boldsymbol{k}=\left(k_{x}, k_{y}\right)$ is the wave vector, which is periodic in the corresponding reciprocal space. The Floquet-Bloch theorem (Eq. (3)) allows to restrict the analysis of an infinite periodic medium to that of a representative unit cell only with Bloch periodic conditions at the unit cell boundaries:

$$
\boldsymbol{u}(x+n a, y+m a)=\boldsymbol{U}(x, y) \exp \left(k_{x} n a+k_{y} m a\right)
$$

Here $n, m$ are integers equal to 0 or 1 for an appropriate boundary edge. Non-redundant values of $k_{x}, k_{y}$ are located within the unit cell of the reciprocal space - Brillouin zone (Fig. SM1).

The boundary-value problem in Eqs. (SM1)-(SM3) can be re-formulated by substituting relation Eq. (SM2) into Eq. (SM1), resulting in:

$$
G \nabla_{\boldsymbol{k}}^{2} \boldsymbol{U}+\left(K+\frac{G}{3}\right) \nabla_{\boldsymbol{k}}\left(\nabla_{\boldsymbol{k}} \cdot \boldsymbol{U}\right)=-\rho \omega^{2} \boldsymbol{U}
$$


where the exponential term $\exp \left(\mathrm{i} \omega \mathrm{t}+\mathrm{i} \boldsymbol{k}^{T} \cdot \mathbf{x}\right)$ has been divided out $\nabla_{\boldsymbol{k}} \boldsymbol{U}=\nabla U+i \boldsymbol{k} \otimes \boldsymbol{U}$, and $\nabla_{\boldsymbol{k}}^{2}=\nabla_{\boldsymbol{k}} \cdot \nabla_{\boldsymbol{k}}$. The boundary conditions (SM3) are then replaced by continuity periodic conditions for displacements on the unit cell.

Equation (SM4) can be solved numerically using the Finite-Element Method (FEM), which allows the analysis of an arbitrarily complex unit cell configuration. The standard Galerkin discretization procedure yields a wave dispersion equation:

$$
\left(\boldsymbol{K}-i\left(k_{x} \boldsymbol{K}_{\mathbf{1}}+k_{y} \boldsymbol{K}_{\mathbf{2}}\right)+k_{x} k_{y} \boldsymbol{K}_{\mathbf{3}}+k_{x}^{2} \boldsymbol{K}_{\mathbf{4}}+k_{y}^{2} \boldsymbol{K}_{\mathbf{5}}-\omega^{2} \boldsymbol{M}\right) \boldsymbol{d}=\mathbf{0}
$$

or, in shorter form:

$$
\left(\boldsymbol{K}_{\boldsymbol{k}}(\boldsymbol{k})-\omega^{2} \boldsymbol{M}\right) \boldsymbol{d}=\mathbf{0}
$$

where $\boldsymbol{K}$ and $\boldsymbol{M}$ are standard stiffness and mass matrices, and $\mathbf{d}$ is the vector of nodal displacements (see, e.g. [3, 4] for further details) The definitions of $\boldsymbol{K}_{i}$ matrices are provided in [3]. The values of d are periodic on the unit cell boundaries. The solution procedure to Eq. (SM6) has been implemented in the Matlab-based code and verified by directly solving Eqs. (SM1), (SM3) by means of a modal analysis procedure with the commercial software COMSOL Multiphysics 4.3 [5]. The representative unit cells are discretized into finite elements by using quadrilateral and triangular elements, respectively. The evaluation of eigenfrequencies $\omega$ has been performed for values of the wave vector $\boldsymbol{k}$ along the borders of the irreducible Brillouin zone, allowing the construction of the corresponding dispersion diagrams (details in the next section).

For a viscoelastic medium, the displacement components cannot be represented as a combination of time- and space-dependent functions by using the Floquet-Bloch theorem in Eq. (SM2). Due to the energy dissipation, spatial wave profiles are time (frequency)-dependent. Also, material characteristics of the medium, $K_{v e}$ and $G_{v e}$, are time-dependent, i.e. $K_{v e}=K_{v e}(t)$ and $G_{v e}=G_{v e}(t)$ 
and are known as volumetric and shear relaxation moduli, respectively. In the frequency domain, the Floquet-Bloch theorem for a periodic viscoelastic medium takes the form:

$$
\widehat{\boldsymbol{u}}(\boldsymbol{x}, \boldsymbol{k}, i \omega)=\widehat{\boldsymbol{U}}(\boldsymbol{x}, \boldsymbol{k}, i \omega) e^{i \boldsymbol{k}^{T} \cdot \boldsymbol{x}}
$$

Here, a hat symbol over the variables indicates the transformed displacements in the frequency domain. The reformulated wave Eq. (SM4) can then be written as follows:

$$
\widehat{G}(\omega) \nabla_{\boldsymbol{k}}^{2} \widehat{\boldsymbol{U}}+\left(\widehat{K}(\omega)+\frac{\hat{G}(\omega)}{3}\right) \nabla_{\boldsymbol{k}}\left(\nabla_{\boldsymbol{k}} \cdot \widehat{\boldsymbol{U}}\right)=-\rho \omega^{2} \widehat{\boldsymbol{U}}
$$

where the dependence of vector $\widehat{\boldsymbol{U}}$ on coordinate vector $\boldsymbol{x}$, wave vector $\boldsymbol{k}$, and frequency $\omega$ is implied and omitted for clarity. Similarly to the previously considered elastic case, Eq. (SM8) can be solved numerically for a representative unit cell subject to continuity periodic conditions for displacements $\widehat{\boldsymbol{U}}$ at the unit cell boundaries.

By using the Galerkin approach, the discretized version of Eq. (SM8) can be derived:

$$
\left(\widehat{\boldsymbol{K}}_{\boldsymbol{k}}(\boldsymbol{k}, \omega)-\omega^{2} \widehat{\boldsymbol{M}}\right) \hat{\mathbf{d}}=\mathbf{0}
$$

Here, similarly to Eq. (SM6), $\widehat{\boldsymbol{K}}_{\boldsymbol{k}}$ and $\widehat{\boldsymbol{M}}$ are the transformed $\boldsymbol{k}$-dependent stiffness and standard mass matrices of the same form as in Eq. (SM6), $\widehat{\boldsymbol{d}}$ is the transformed vector of nodal displacements. However, contrary to Eq. (SM6) and Eq. (SM9), here the stiffness matrix $\widehat{\boldsymbol{K}}_{k}$ is frequencydependent. This dependence does not allow to evaluate eigenfrequencies directly by solving Eq. (SM9). However, this issue can be overcome by inverting the problem, e.g. by specifying the value of $\omega$ and formulating an eigenvalue problem for wave vector $\boldsymbol{k}$. Since the wave vector has two unknown components, it is necessary to introduce a relation between $k_{x}$ and $k_{y}$ in order to derive a typical eigenvalue problem.

Thus, by assuming e.g. $k_{y}=c k_{x}$ with $c$ a real constant and $0<c<1$, Eq. (SM9) can be rewritten as [4]:

$$
\left(\breve{\boldsymbol{K}}_{\boldsymbol{k}}(\omega)-k_{x} \widetilde{\boldsymbol{M}}_{\boldsymbol{k}}\right) \breve{\boldsymbol{d}}_{\boldsymbol{k}}=0
$$


where

$$
\breve{\boldsymbol{K}}_{\boldsymbol{k}}=\left[\begin{array}{cc}
-i\left(\boldsymbol{K}_{1}+c \boldsymbol{K}_{2}\right) & \boldsymbol{K}-\omega^{2} \boldsymbol{M} \\
\boldsymbol{I} & \mathbf{0}
\end{array}\right], \breve{\boldsymbol{M}}_{\boldsymbol{k}}=\left[\begin{array}{cc}
-\left(\boldsymbol{K}_{4}+c \boldsymbol{K}_{3}+c^{2} \boldsymbol{K}_{5}\right) & \mathbf{0} \\
\mathbf{0} & \boldsymbol{I}
\end{array}\right], \quad \breve{\boldsymbol{d}}_{\boldsymbol{k}}=\left[\begin{array}{c}
k_{x} \boldsymbol{d} \\
\boldsymbol{d}
\end{array}\right] .
$$

For any specified value of $\omega$, Eq. (SM10) is an eigenvalue problem for $k_{x}$ with the continuity periodic conditions for $\widetilde{\boldsymbol{d}}_{\boldsymbol{k}}$ at the unit cell boundary. Since most of the available FEM software does not allow to easily modify the element stiffness and mass matrices, the numerical solution procedure for Eq. (SM6) has been implemented as a Matlab-based code. For a periodic viscoelastic medium, all the eigenvalues $k_{x}$ are complex-valued with the imaginary part describing spatially attenuated waves [3].

The evaluation of the wave dispersion for a viscoelastic metamaterial is performed by first fixing the value of $c$ in $k_{y}=c k_{x}$ equal to 0 or 1 , which corresponds to the $\Gamma X$ or $\Gamma M$ borders of the irreducible Brillouin zone, respectively, and then solving the eigenvalue problem in Eq.(11) for the specific frequencies in the relevant frequency range. Finally, the real parts of the derived solutions $k_{x}$ are plotted versus the frequencies as common two-dimensional dispersion diagrams. The imaginary parts of $k_{x}$ are used to evaluate the wave attenuation level $\xi=2 \operatorname{Im}\left(k_{x}\right) / \operatorname{Re}\left(k_{x}\right)$, thus providing the attenuation spectrum.

To validate attenuation capabilities of the metamaterial structures, we additionally performed transmission analysis on finite-size metastructures with COMSOL Multiphysics 4.3 [6]. The calculations are carried out in the frequency domain for structures composed of 8 adjacent unit cells for the hub-spoke geometry and 4 adjacent unit cells for the cross-like cavity geometry, arranged as a $1 \mathrm{D}$ array in the horizontal direction. The structures are modelled as infinite in the vertical direction by applying continuity periodic boundary conditions for wave displacements at the top and bottom boundaries. The analysis is performed using horizontally-polarized plane waves incident on one boundary of the structure to model waves propagating along the $\Gamma X$ direction. The opposite 
boundary is subject to non-reflecting boundary conditions (Perfectly Matched Layers - PML - the size of 5 unit cells) to eliminate reflections of the incident wave field. The frequency is swept in 500 or 1000 steps within the ranges specified for each case under consideration from the corresponding dispersion diagrams. The transmitted wave field $T$ is evaluated as:

$$
T=\log \int_{A}\left|\frac{\boldsymbol{u}_{t r}}{\boldsymbol{u}_{\text {in }}}\right| d A
$$

where $\boldsymbol{u}_{\text {in }}$ is the incident displacement amplitude, $\boldsymbol{u}_{t r}$ is the transmitted amplitude, and $A$ is the analysed area of 5 unit cells of homogeneous material after the metamaterial region.

\section{Evaluation of the dispersion spectra: direct and reciprocal periodic lattices}

In this work, dispersion of elastic waves in metamaterials is studied numerically by analyzing twodimensional periodic structures with a square lattice of infinite extent. The dynamic properties of such structures can be characterized by considering a primitive unit cell, which is a minimum unit representing the whole structure by its periodic repetition. Figure SM1 shows one of the studied metamaterial configurations with a primitive unit cell, and the lattice vectors $\mathbf{a}_{1}, \mathbf{a}_{2}$ of equal length $a$. This lattice is known as a direct lattice.

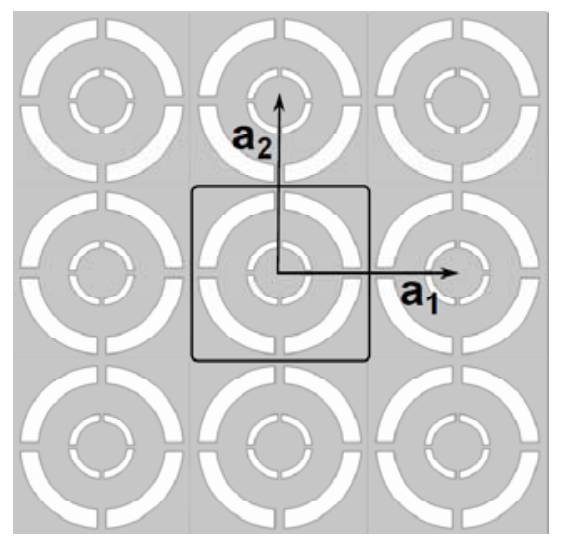

(a)

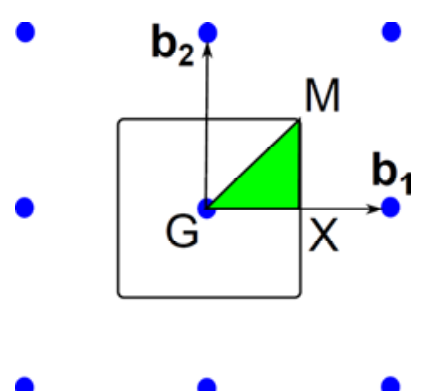

(b)

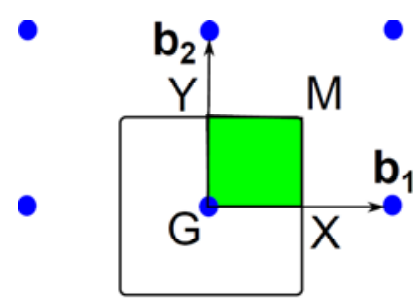

(c) 
Figure SM1. a) Periodic hierarchical metamaterial structure with a square lattice (direct lattice); b) reciprocal lattice corresponding to the direct lattice with mirror-reflection symmetry along vertical, horizontal and diagonal directions; c) reciprocal lattice corresponding to direct lattice with mirrorreflection symmetry along only vertical and horizontal directions.

Reciprocal lattice is specified by the lattice vectors $\mathbf{b}_{1}, \mathbf{b}_{2}$ defined as follows:

$$
\mathbf{a}_{\mathrm{i}} \cdot \mathbf{b}_{\mathbf{j}}=2 \pi \delta_{i j} \text {, or } \mathbf{b}_{1}=\frac{2 \pi}{\mathbf{a}_{1}}, \mathbf{b}_{2}=\frac{2 \pi}{\mathbf{a}_{2}}
$$

with $\delta_{i j}$ the Kronecker delta. Thus, the square reciprocal lattice is also spatially periodic with the period $1 / a$. Hence, one only needs to consider $\omega(\mathbf{k})$ for non-redundant vectors $\mathbf{k}$ located within the first Brillouin zone, which is indicated by black squares in Fig. SM1b-c [7]. Moreover, this domain can be further truncated to the irreducible Brillouin zone by taking into account symmetries of rotation, mirror-reflection and time-inversion. If, besides time-inversion, a metamaterial has mirrorreflection symmetries along the direct lattice vector lines as well as along the diagonal of a unit cell, the irreducible Brillouin zone is a triangle indicated by green in Fig. SM1b. If the mirror-reflection symmetry along the diagonals is absent, the irreducible Brillouin zone is a rectangle shown by green in Fig. SM1c.

\section{Parametric study}

The results for the hub-spoke metamaterial discussed in the main text show that introduction of the hierarchical organization leads to a reduction of the width of a lower wide BG compared to that of the corresponding regular configuration. We perform here parametric studies on the regular and hierarchical structures to determine the geometries that open BGs more efficiently. In the case of the 
regular configuration (Fig. SM2), the BG size is almost independent on the thickness of the connecting elements and the mass of the internal inclusion.
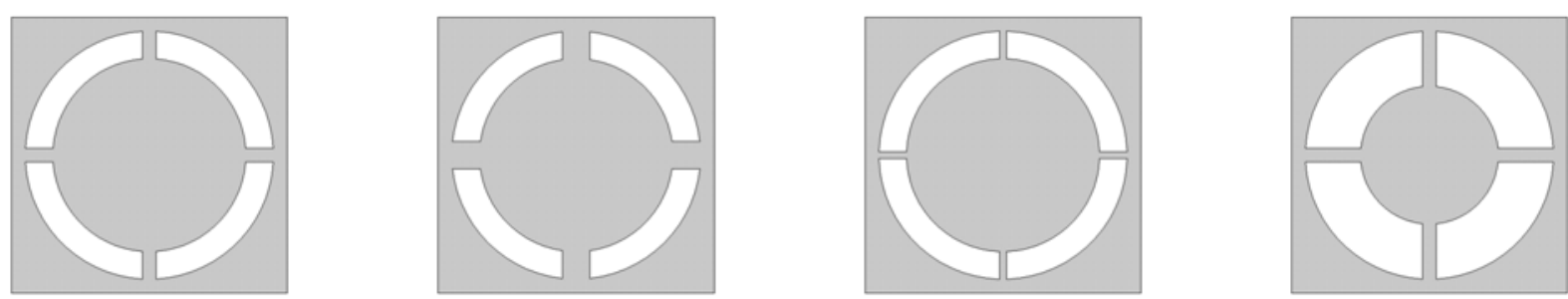

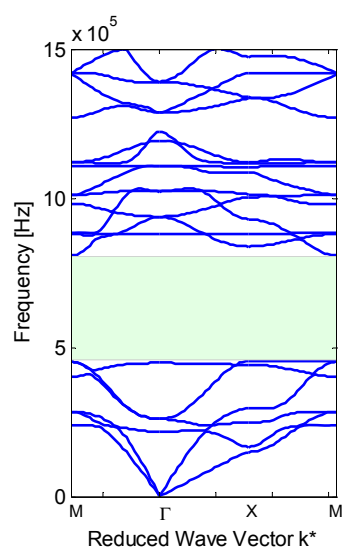

(a)

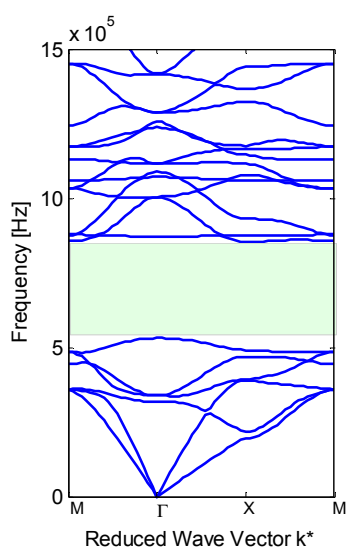

(b)

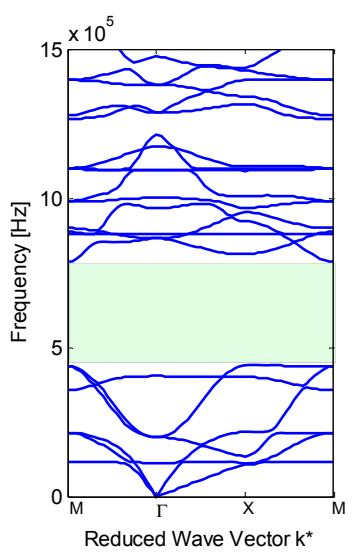

(c)

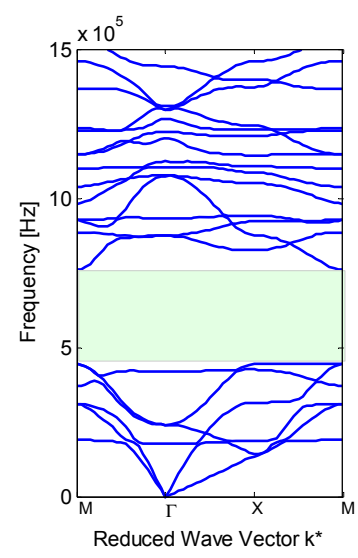

(d)

Figure SM2. Dispersion diagrams for regular metamaterial unit cells with a) the same geometric parameters as in the main text (reference case); b) two times thicker connectors $\left(d_{2}=0.1 a_{2}\right)$ and c) two times thinner connectors $\left(d_{2}=0.025 a_{2}\right)$ than in the reference case, $\left.d\right)$ smaller internal mass ( $\left.c_{2}=0.25 a_{2}\right)$ compared to the reference case. The BG size is almost the same in all the cases.

By introducing the hierarchical organization, the BG separates into a lower BG, which is approximately of at the same frequency as that shown in Fig. SM2, and one or more higher BGs, whose width and frequency range can be modified by changing the geometry of the nested structure (see Fig. SM3). A self-similar structure (Fig. SM3a) preserves a similar lower BG, with additional narrow higher-frequency BGs. 


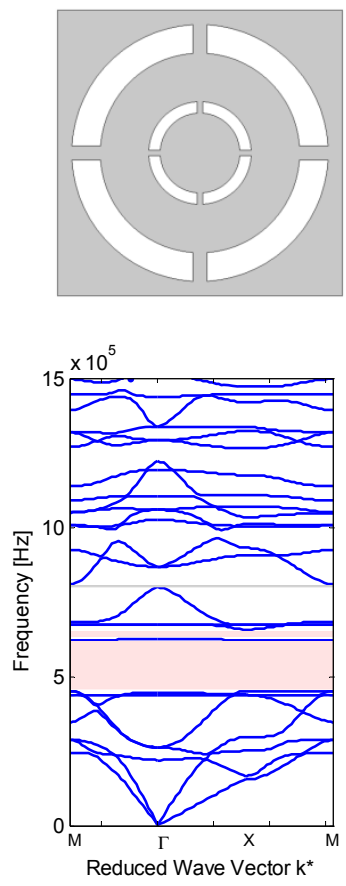

(a)
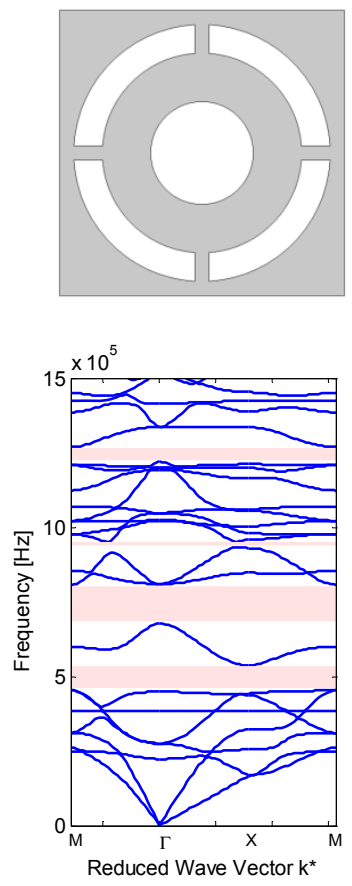

(b)
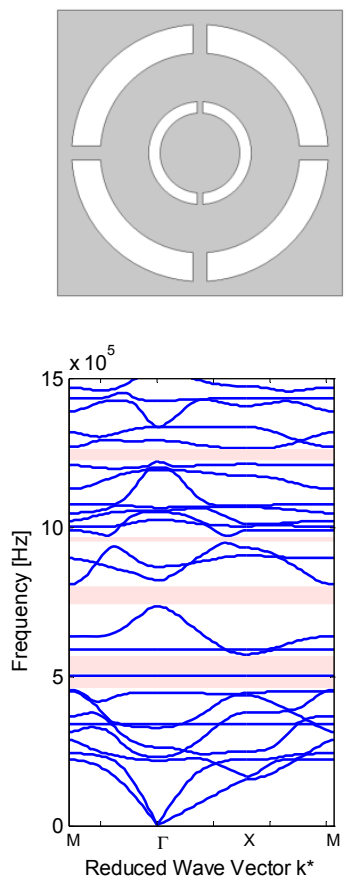

(c)
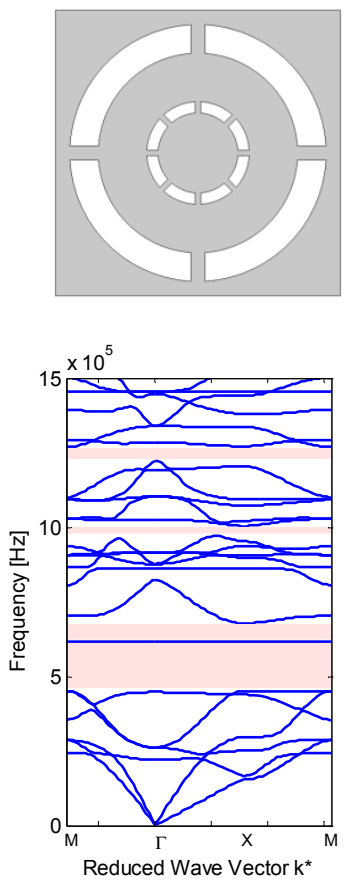

(d)

Figure SM3. Dispersion diagrams for the hierarchical unit cells with a) self-similar and b-d) nonself-similar hierarchical levels.

\section{Equivalent spring-mass model for a metamaterial with cross-like cavities}

Wang et al. [8] revealed that the lowest BG for a metamaterial with cross-like cavities is induced due to the local resonance of masses formed by edges of four unit cells (see Fig. SM4a) connected by thin ligaments acting as springs. Based on this, they proposed an equivalent mass-spring model capable of predicting the lower bound of the BG. This model can be extended to hierarchically organized metamaterials. 
(a)

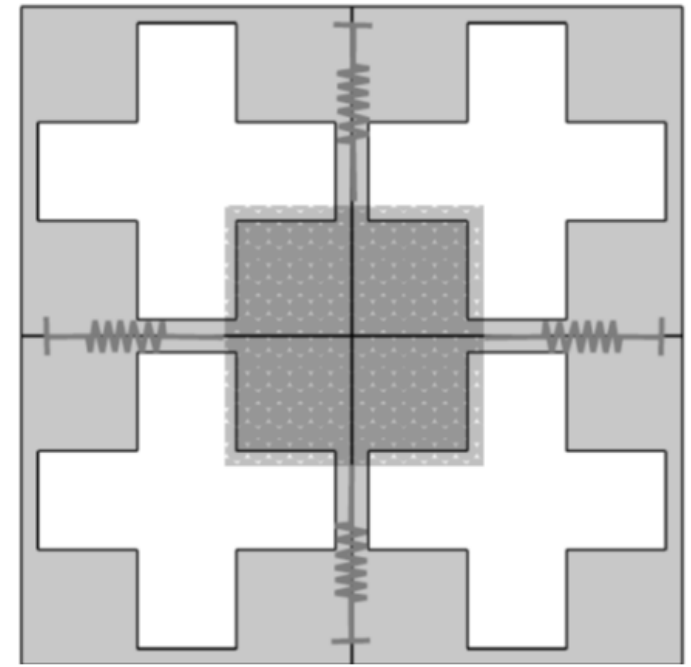

(b)

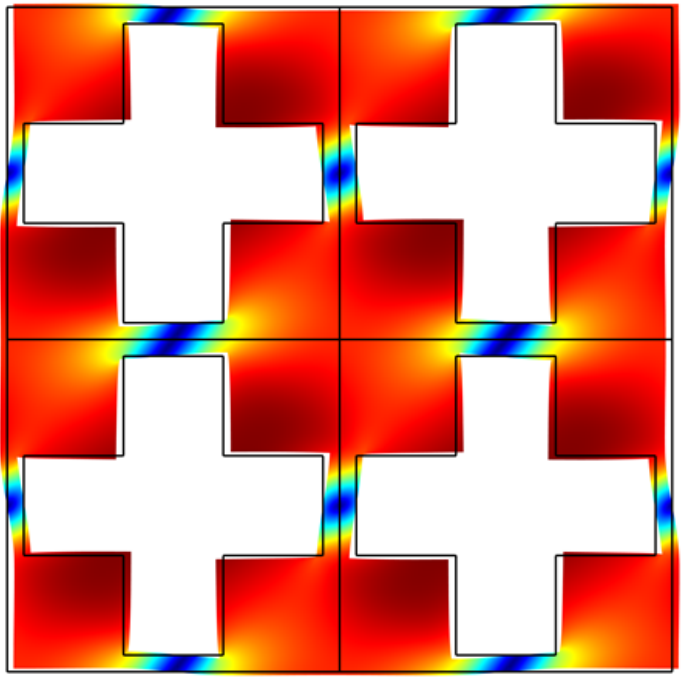

Figure SM4. (a) Four adjacent unit cells of the metamaterial with cross-like cavities and an equivalent spring-mass model capable of evaluated the lower bound of the $1^{\text {st }} B G$ developed in Ref. [8] capable of evaluating the lower bound of the $1^{\text {st }} B G$. (b) The vibration pattern of the lower bound of the $1^{\text {st }} B G$.

\section{Hierarchical metamaterial with L-like cavities}


To further substantiate the generality of the findings regarding hierarchical metamaterials, in this section we consider a new structure, the unit cell of which displays an L-like cavity (see Fig. SM5a). Material parameters are the same as those used in the main paper while the geometrical parameters are: $a=1 \mathrm{~mm}, b=0.9 \cdot a$ and $c=0.1 \cdot a$. A hierarchical factor of $\mathrm{HF}=10$ is used, leading to the final hierarchical geometry represented in Fig. SM5b.

As expected, the same physics described in Section 3.1 of the Main text is observed, i.e., the two BGs ranging from approximately 298 to $315 \mathrm{kHz}$ and 469 to $578 \mathrm{kHz}$ in Fig. SM5c (relative to the ordinary structure) are largely preserved in the diagram of Fig. SM5d (relative to the hierarchical structure). For example, some pass bands located inside the latter BG are present but characterized by mostly flat lines corresponding to localized modes (Fig. SM5e-top). As discussed in the main paper, these modes become non-propagating in real structures, which always possess a certain level of energy dissipation. Here, for the sake of clarity the computations are limited only to the linear elastic case (refer to the main paper for an estimation of the effect of viscoelasticity).

As for the case presented in the main paper, it can be seen that the introduction of hierarchy allows to open a low-frequency BG in a more lightweight structure (refer to Section 3.1 of the main text for further details). 


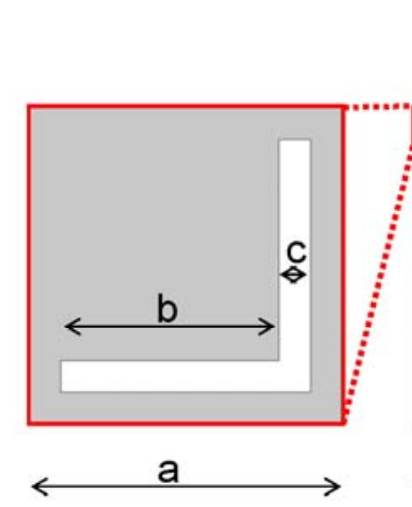

(a)

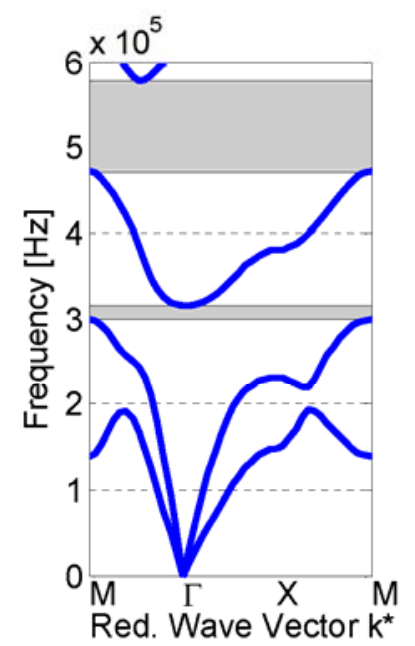

(c)

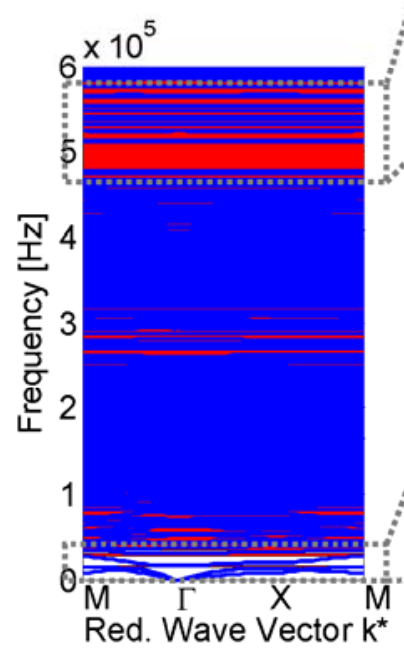

(d)

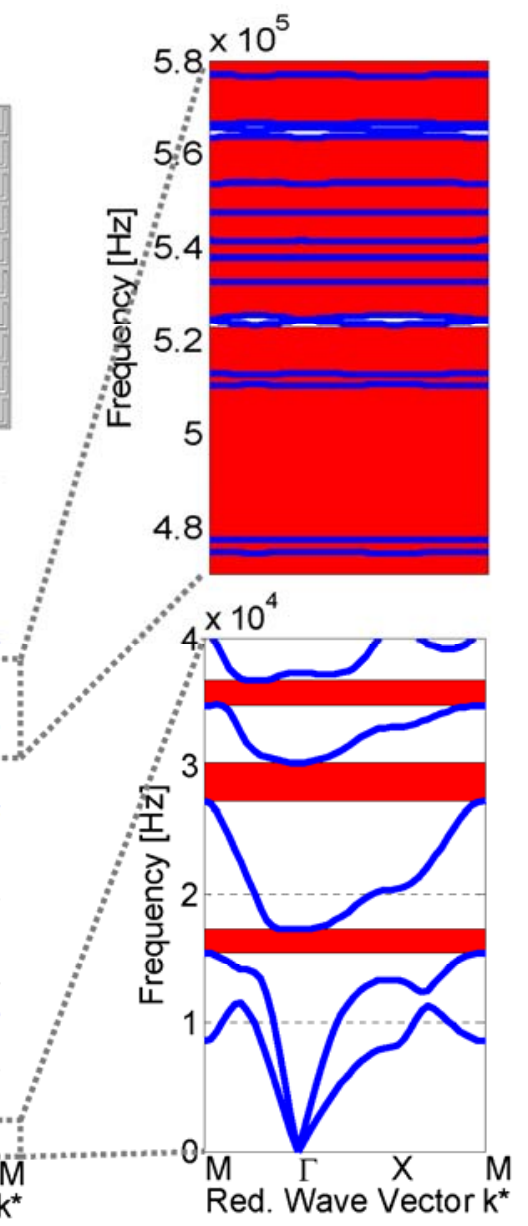

(e)

Figure SM5: Unit cell for an L-like porous metamaterial with (a) regular and (b) external hierarchical organization and (c-d) their corresponding dispersion diagrams. (e) Two zooms of portions of the dispersion diagram for the hierarchical metamaterial.

\section{Comparison between a simple unit cell scaling and a hierarchical metamaterial}

To better emphasize the mechanisms specifically involved with structural hierarchy, in this Section we compare the variation of the dispersion spectra in two scenarios:

(i) introducing simple scaling of the unit cell 
(ii) introducing a scaling of the unit cell with the additional introduction of a self-similar hierarchy at different scale levels

We consider several systems with $N \times N(N=1,2, \ldots, 20)$ unit cells, as shown in Fig. SM6a-c. From the schematic representation the lattice parameter can be recursively evaluated as $a / N$ (with $N=1,2$, 20 and $\mathrm{a}=1 \mathrm{~mm}$ ) for each configuration. At each iteration, the unit cell required for a proper application of the Bloch-Floquet conditions is highlighted by a red square. Figures SM6d-f report the corresponding dispersion diagrams for these systems. Comparing them, the dispersion diagrams in the case of "simple scaling" are exactly the same but scaled towards higher frequencies (the y-axis upper limit is shifted from 1.5 MHz in Fig. SM6d to $30 \mathrm{MHz}$ in Fig. SM6f), proportionally with to the size reduction of the lattice parameter. No new dispersion curves and no new BGs can be observed.

On the contrary, in the case of the unit cell with added hierarchy (see the main text of the paper), a much richer phenomenology is present. This includes many more dispersion curves in the same frequency range, dispersion diagram self-similarity at very low frequencies, nucleation of new (lowfrequency) BGs, localized modes, etc.

This allows us to say that the topological hierarchical architecture can be effectively used to tune BGs, dispersion curves and localize motion in preselected regions of the unit cell. 
a)

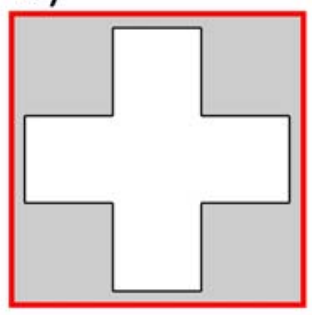

$1 \times 1$

d)

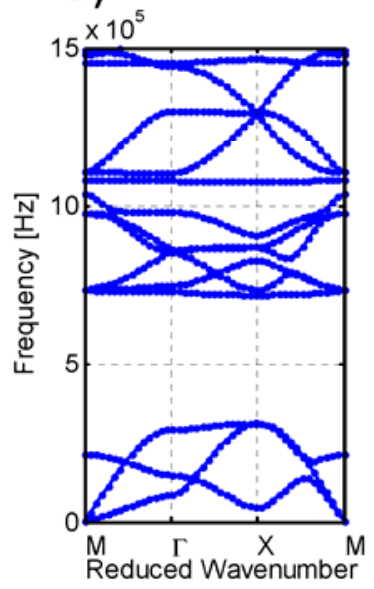

b)

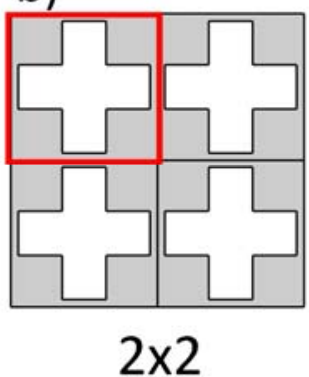

e)

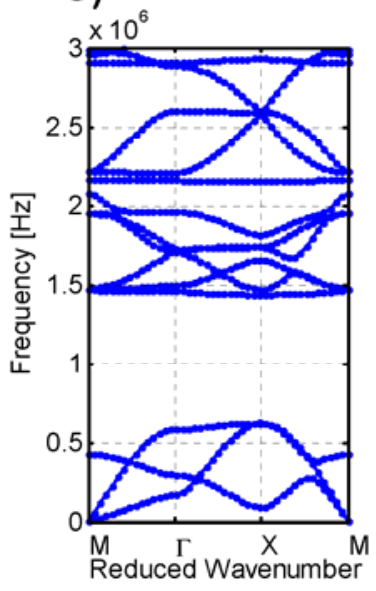

c)

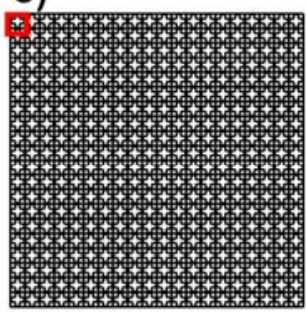

$\mathrm{NxN}$

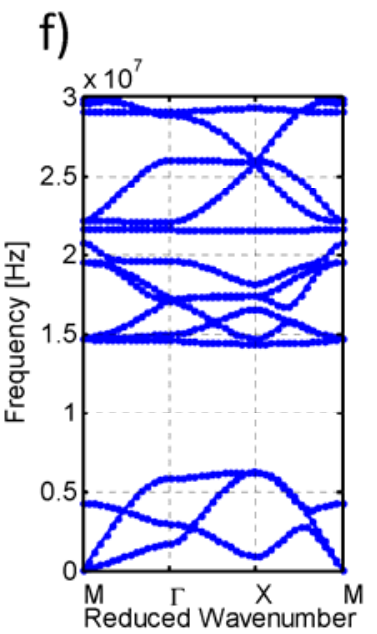

Figure SM6: Schematic representation of (a) 1x1, (b) $2 x 2$ and (c) NxN unit cell systems. The lattice parameter for each configuration can be evaluated as a/N (with $N=1,2$ and 20) and (d-f) their respective dispersion diagrams. The unit cells for the Bloch analysis are highlighted with a red rectangle.

\subsection{Numerical analysis of samples for experimental tests}

The sample considered in experiments is shown in Fig. SM7. In this case, the dispersion diagram is predicted numerically using a full 3D model to capture all possible wave modes propagating in the structure. Cells are meshed by means of 4-node tetrahedral elements of maximum size $1 \mathrm{~mm}$ in order to provide accurate eigensolutions up to the maximum frequency of interest ( $300 \mathrm{kHz})$. The band structure is derived assuming periodic (in the $x$-direction) and free (in the $y$-direction) 
boundary conditions at the edges of the cell domain. Free boundary conditions are imposed at the top and bottom surfaces of the cell. Numerically calculated mode shapes are shown in Fig. SM 8

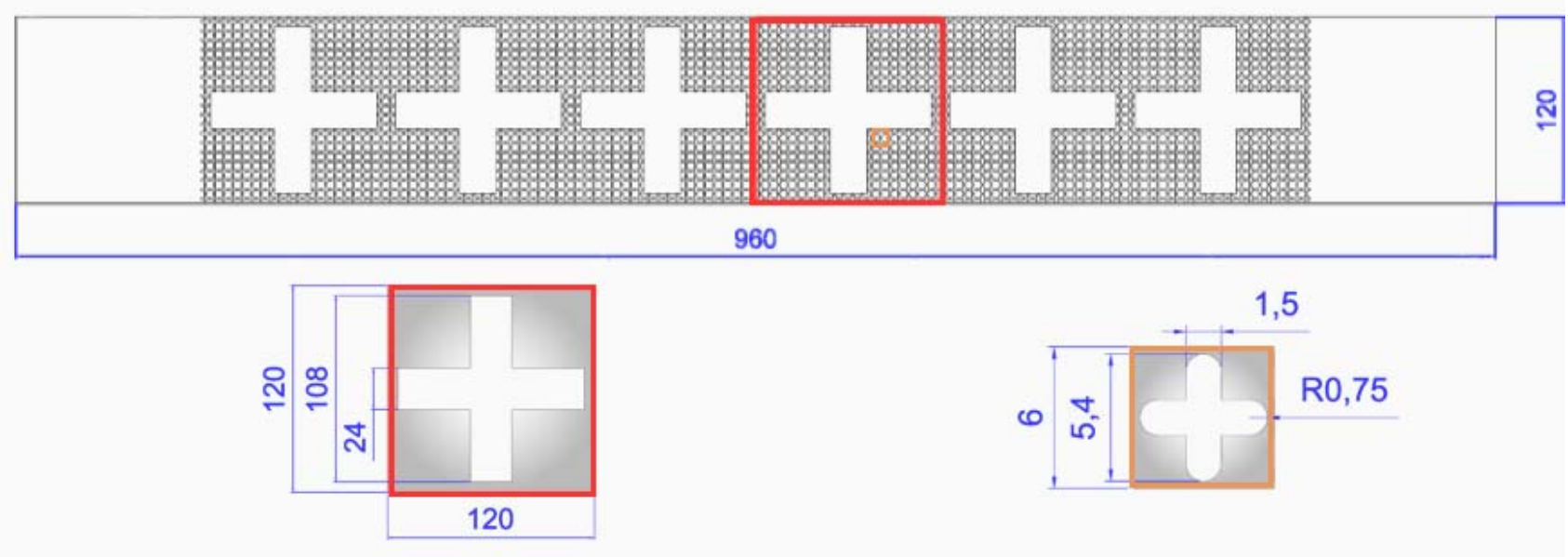

Figure SM7: Geometry and dimensions (in $\mathrm{mm}$ ) of the experimental sample. Highlighted in red and orange are the large and small cross-like cavity unit cells, respectively.
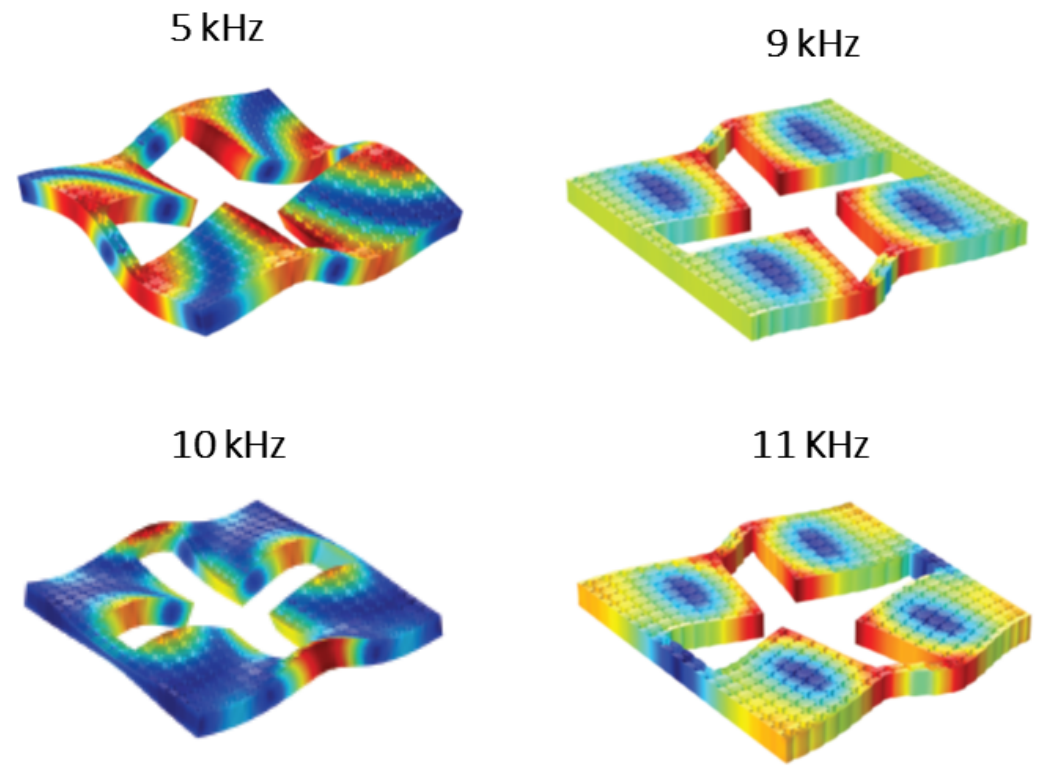

Figure SM8: Numerically calculated mode shapes for the hierarchical-cross-like cavity unit cells in the low frequency range. 

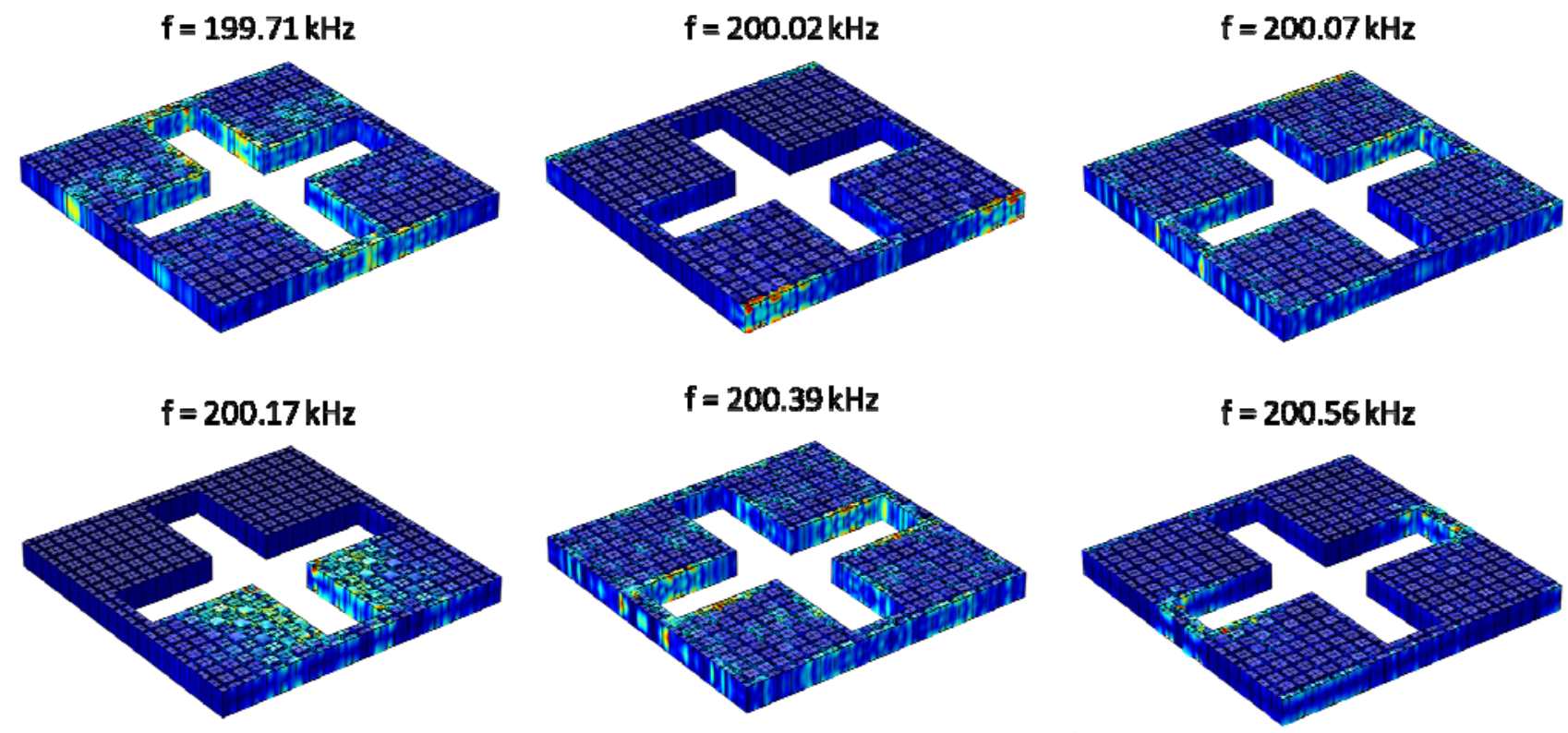

Figure SM9: Numerically calculated mode shapes for the hierarchical-cross-like cavity unit cells in the high frequency range.

\section{References}

[1] D. Royer and E. Dieulesaint, Elastic waves in solids, New-York: Wiley, 1999.

[2] L. Brillouin, Wave propgataion in periodic structures, New York: Dover, 1946.

[3] A. Krushynska, V. Kouznetsova and M. Geers, "Visco-elastic effects on wave dispersion in three-phase acoustic metamaterials," J. Mech. Phys. Solids, vol. 96, pp. 29 - 47, 2016.

[4] E. Andreassen and J. Jensen, "Analysis of phononic bandgap structures with dissipation," ASME J. Vibr. Acoust., vol. 135, p. 041015, 2013.

[5] M. Miniaci, A. Marzani, N. Testoni and L. De Marchi, "Complete band gaps in a polyvinyl chloride (PVC) phononic plate with cross-like holes: numerical design and experimental verification," Ultrasonics, vol. 56, pp. $251-259,2015$.

[6] Comsol Multiphysics, www.comsol.com, Release 4.3a.

[8] Y.-F. Wang, W. Y.-S. and X.-X. Su, "Large bandgaps of two-dimensional phononic crystals with cross-like holes," J. Appl. Phys., vol. 110, p. 113520, 2011. 